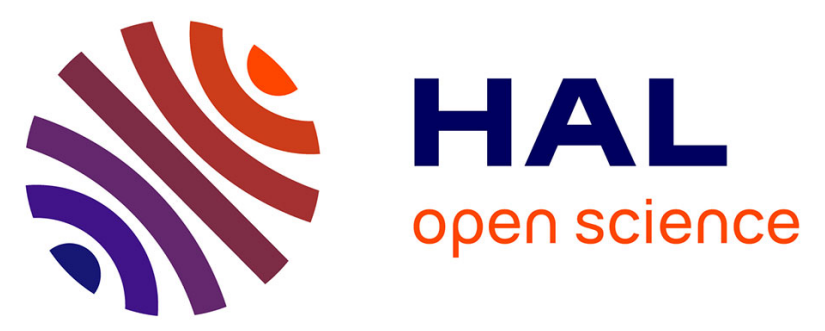

\title{
Steatosis and gut microbiota dysbiosis induced by high-fat diet are reversed by 1-week chow diet administration
}

Zahra Safari, Magali Monnoye, Peter M. Abuja, Mahendra Mariadassou, Karl Kashofer, Philippe Gerard, Kurt Zatloukal

\section{To cite this version:}

Zahra Safari, Magali Monnoye, Peter M. Abuja, Mahendra Mariadassou, Karl Kashofer, et al.. Steatosis and gut microbiota dysbiosis induced by high-fat diet are reversed by 1-week chow diet administration. Nutrients, 2019, 71, pp.72-88. 10.1016/j.nutres.2019.09.004 . hal-02503316

\section{HAL Id: hal-02503316 https://hal.science/hal-02503316}

Submitted on 9 Mar 2020

HAL is a multi-disciplinary open access archive for the deposit and dissemination of scientific research documents, whether they are published or not. The documents may come from teaching and research institutions in France or abroad, or from public or private research centers.
L'archive ouverte pluridisciplinaire HAL, est destinée au dépôt et à la diffusion de documents scientifiques de niveau recherche, publiés ou non, émanant des établissements d'enseignement et de recherche français ou étrangers, des laboratoires publics ou privés.

\section{(c)(1)}

Distributed under a Creative Commons Attribution| 4.0 International License 


\title{
Steatosis and gut microbiota dysbiosis induced by high-fat diet are reversed by 1-week chow diet administration
}

\author{
Zahra Safari $^{a, b}$, Magali Monnoye ${ }^{b}$, Peter M. Abuja ${ }^{a}$, Mahendra Mariadassou ${ }^{c}$, \\ Karl Kashofer ${ }^{a}$, Philippe Gérard ${ }^{b, *}$, Kurt Zatloukal ${ }^{a, * *}$ \\ a Institute of Pathology, Medical University of Graz, 8010 Graz, Austria \\ ${ }^{\mathrm{b}}$ Micalis Institute, INRA, AgroParisTech, Université Paris-Saclay, 78350 Jouy-en-Josas, France \\ ${ }^{\mathrm{c}}$ MaIAGE, UR1404, INRA, 78350 Jouy-en-Josas, France
}

\section{A R T I C L E I N F O}

Article history:

Received 24 April 2019

Revised 26 August 2019

Accepted 13 September 2019

\section{Keywords:}

Reversibility

Microbiome

Liver steatosis

NAFLD

High-fat diet

Diet switch

\begin{abstract}
A B S T R A C T
Many studies have recently shown that diet and its impact on gut microbiota are closely related to obesity and metabolic diseases including nonalcoholic fatty liver disease. Gut microbiota may be an important intermediate link, causing gastrointestinal and metabolic diseases under the influence of changes in diet and genetic predisposition. The aim of this study was to assess the reversibility of liver phenotype in parallel with exploring the resilience of the mice gut microbiota by switching high-fat diet (HFD) to chow diet (CD). Mice were fed an HF for 8 weeks. A part of the mice was euthanized, whereas the rest were then fed a CD. These mice were euthanized after 3 and 7 days of feeding with $C D$, respectively. Gut microbiota composition, serum parameters, and liver morphology were assessed. Eight weeks of HFD treatment induced marked liver steatosis in mice with a perturbed microbiome. Interestingly, only 7 days of CD was enough to recover the liver to a normal status, whereas the microbiome was accordingly reshaped to a close to initial pattern. The abundance of some of the bacteria including Prevotella, Parabacteroides, Lactobacillus, and Allobaculum was reversible upon diet change from HFD to CD. This suggests that microbiome modifications contribute to the metabolic effects of HFD feeding and that restoration of a normal microbiota may lead to improvement of the liver phenotype. In conclusion, we found that steatosis and gut microbiota dysbiosis induced by 8 weeks of high-fat diet can be reversed by 1 week of chow diet administration, and we identified gut bacteria associated with the metabolic phenotype.
\end{abstract}

(c) 2019 Elsevier Inc. All rights reserved.
Abbreviations: CD, chow diet; H\&E, hematoxylin and eosin; HFD, high-fat diet; NAFLD, nonalcoholic fatty liver disease; TG, triglyceride.

* Corresponding author. Tel.: +3313465 2428.

E-mail addresses: philippe.gerard@inra.fr (P. Gérard), kurt.zatloukal@medunigraz.at (K. Zatloukal).

\section{Introduction}

Nonalcoholic fatty liver disease (NAFLD) comprises a spectrum of disease ranging from simple and usually nonprogressive steatosis to nonalcoholic steatohepatitis (NASH) [1,2]. Obesity and insulin resistance have been identified as major NAFLD risk factors [3]. NAFLD is associated with altered hepatic lipid metabolism leading to fat deposition and de novo lipid synthesis in the liver. In recent years, evidences suggest an 
involvement of the microbiota in NAFLD development [4]. In an early study, Drenick et al [5] observed hepatic steatosis development in patients undergoing gastric bypass surgery that coincided with bacterial overgrowth. Subsequently, small intestinal bacterial overgrowth has been shown to be more prevalent in patients with NASH than in healthy controls [6]. Later, Henao-Mejia et al [7] provided evidence that modulation of the intestinal microbiota through multiple inflammasome components is a critical determinant of NAFLD and NASH progression, whereas it has been demonstrated the gut microbiota protects against steatohepatitis in the methioninecholine-deficient model [8]. Using microbiota transplants strategy, we have further revealed that the propensity to develop NAFLD directly depends on gut microbiota composition, independently of obesity [9]. Altogether, these findings suggest that high-fat diet (HFD) alone is not sufficient to cause obesity or NAFLD development and that the gut microbiota contributes to these diet-induced metabolic disorders.

Dietary lipids have been linked to the development of hepatic steatosis and NASH in both animal models and humans; however, their role in pathophysiology of NAFLD remains unclear [10-12]. In the liver, an HFD leads to exaggerated free fatty acid levels, which induce hepatic insulin resistance, decreased fatty acid oxidation, and de novo lipogenesis in hepatocytes, causing weight gain and hepatic steatosis [13]. Lieber et al [14] used an HFD in male SpragueDawley rats and demonstrated the development of steatosis within 3 weeks associated with insulin resistance and increased markers of fibrogenesis. Similar results were reported subsequently in male C57BL/6 mice fed with an HFD for up to 16 weeks. HFD-fed mice demonstrated an increase in body weight, steatosis development, hepatocyte ballooning, augmented fasting serum glucose levels, and reduced adiponectin levels, suggesting hyperglycemia and insulin resistance [15]. Therefore, HFD treatments of rodents are commonly used as obesity and NAFLD models and are considered closer to human pathologies than models of gene inactivation. This is important because of the growing obesity epidemic [16] and the importance of overnutrition in the development of diseases, such as the metabolic syndrome and NAFLD [17-19].

Dietary changes have also been demonstrated to be central drivers of microbiome composition and function, and mice fed HFD have different microbiota composition from those that have been fed control or balanced diets [20,21]. Moreover, feeding different types of fat results in very different compositions of gut microbiota in mice. Similarly, the highfat/high-sugar diet associated with Western lifestyle has altered the composition and metabolic activity of the human gut microbiome [22]. Such diet-induced changes to gutassociated microbial communities are now suspected of contributing to growing epidemics of chronic illness in the developed world, including obesity $[23,24]$ and NAFLD $[7,9,25]$.

Animal and human studies have shown the reversibility of NAFLD [26-28]. Indeed, in mild forms, fatty liver can be a reversible condition that could be improved with lifestyle modifications such as weight loss, diet changes, and increased physical activity. It was also observed that when obese humans changed their diet to restrict fat or carbohydrate, their gut microbiota shifted from an "obese" to a "lean" microbiome composition [23]. However, Thaiss et al [29] have recently discovered that although many of the structural alterations of the microbiome are reversible upon weight normalization after obesity, certain elements of the obesity-associated composition and function persist after dieting even when many of the metabolic parameters have already normalized to preobesity levels.

Therefore, we propose the hypothesis that the effect of diet may be partly mediated through the intestinal microbiome whose products are transported into the liver and from there are transmitted to the rest of the body contributing to disease (or health) $[30,31]$. This implies that targeting the gut bacteria to restore a balanced microbiome could lead to disease improvement. The understanding of this complexity is changing nutrition science particularly related to liver diseases, and it is of considerable interest to decipher the reversibility of both NAFLD and gut microbiome dysbiosis and how they are related. Therefore, the objective of this study was to investigate to what extend the HF-induced steatosis and dysbiosis can be reversed by a chow diet and to identify which gut bacteria could be involved in disease development.

\section{Methods and materials}

\subsection{Animal experiments}

The experiments were carried out with male C57BL/6J mice obtained from Charles River (Sulzfeld, Germany) at the age of 8 weeks. The animals were housed 4 per cage under controlled conditions of temperature (set point $21^{\circ} \mathrm{C}$ ) and air humidity (set point 50\%) and under a 12-hour light/dark cycle. The bedding of the cages was mixed, and mice were allowed to acclimate for 2 weeks prior to start of the experiment while being fed a CD. Twelve mice were used for the study. HFD (E15741-347 $\gamma$-irradiated $25 \mathrm{kGy}$ ) and chow diet (control diet, CD; V1534-703 $\gamma$-irradiated $25 \mathrm{kGy}$ ) used in this study were

\section{Table 1 - Ingredient composition of the CD}

\begin{tabular}{lr} 
Ingredient & Amount $(\mathrm{g} / \mathrm{kg})$ \\
\hline Wheat meal & 220.00 \\
Wheat middlings & 100.00 \\
Wheat feed & 180.00 \\
Barley & 160.00 \\
Oat hulls and bran & 31.00 \\
Soybeans/soybean meal (heat treated/toasted) & 215.00 \\
Soybeans concentrate (heat treated/toasted) & 12.50 \\
L-Lysine HCl & 1.00 \\
LL-Methionine & 0.30 \\
Vitamin and trace element premix with cereals & \\
as carrier & 10.00 \\
Choline chloride & 3.20 \\
Salt (NaCl) & 6.00 \\
Calcium phosphate, monobasic & 10.00 \\
Calcium carbonate & 12.50 \\
Calcium propionate & 5.00 \\
Sorbic acid & 0.50 \\
Sugar beet pulp & 33.00 \\
\hline
\end{tabular}

For vitamin mix and trace element composition, see Table 4 . The kJ percentages for fat, protein, and carbohydrate were 9, 24, and 67, respectively. 


\section{Table 2 - Ingredient composition of the HFD}

\begin{tabular}{lr} 
Ingredient & Amount (g/kg) \\
\hline Casein & 277.00 \\
Maltodextrin & 156.00 \\
Sucrose & 84.90 \\
Cellulose powder & 60.00 \\
L-Cystine & 3.50 \\
Vitamin premix & 10.00 \\
Mineral and trace element premix & 60.00 \\
Choline chloride (50\% choline) & 2.50 \\
Butylated hydroxytoluene & 0.10 \\
Beef tallow, premier Jus & 315.00 \\
Soybean oil & 31.00 \\
\hline For vitamin mix and trace element composition, see Table 4. The kJ \\
percentages for fat, protein, and carbohydrate were 60, 20, and 20, \\
respectively.
\end{tabular}

Table 3 - Mineral, fatty acid, and amino acid composition of the CD and HFD

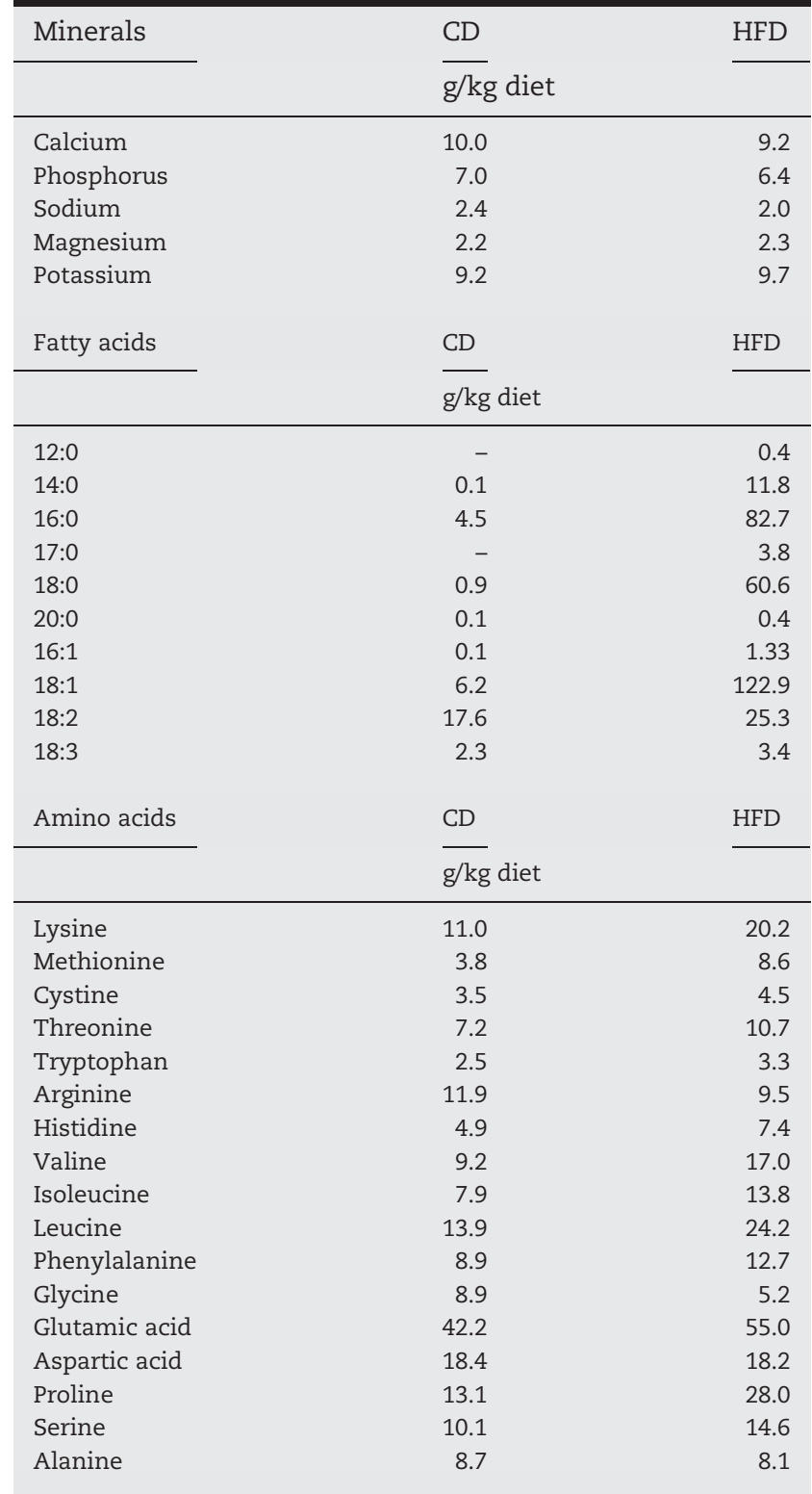

Table 4-Vitamin and trace element composition in the CD and HFD

\begin{tabular}{|c|c|c|}
\hline Vitamins & CD & HFD \\
\hline & per kg diet & \\
\hline Vitamin A & $25000 \mathrm{IU}$ & $15000 \mathrm{IU}$ \\
\hline Vitamin D3 & $1500 \mathrm{IU}$ & $1500 \mathrm{IU}$ \\
\hline Vitamin E & $135 \mathrm{mg}$ & $150 \mathrm{mg}$ \\
\hline Vitamin $\mathrm{K}$ (as MNB) & $20 \mathrm{mg}$ & $20 \mathrm{mg}$ \\
\hline Thiamine (B1) & $86 \mathrm{mg}$ & $25 \mathrm{mg}$ \\
\hline Riboflavin (B2) & $32 \mathrm{mg}$ & $16 \mathrm{mg}$ \\
\hline Pyridoxine (B6) & $31 \mathrm{mg}$ & $16 \mathrm{mg}$ \\
\hline Cobalamin (B12) & $150 \mu \mathrm{g}$ & $30 \mu \mathrm{g}$ \\
\hline Nicotinic acid & $153 \mathrm{mg}$ & $47 \mathrm{mg}$ \\
\hline Pantothenic acid & $59 \mathrm{mg}$ & $55 \mathrm{mg}$ \\
\hline Folic acid & $10 \mathrm{mg}$ & $16 \mathrm{mg}$ \\
\hline Biotin & $710 \mu \mathrm{g}$ & $300 \mu \mathrm{g}$ \\
\hline Choline & $1370 \mathrm{mg}$ & $920 \mathrm{mg}$ \\
\hline \multirow[t]{2}{*}{ Trace elements } & $\mathrm{CD}$ & HFD \\
\hline & $\overline{\mathrm{mg} / \mathrm{kg} \text { diet }}$ & \\
\hline Iron & 186 & 168 \\
\hline Manganese & 68 & 95 \\
\hline Zinc & 91 & 65 \\
\hline Copper & 15 & 13 \\
\hline Iodine & 2.1 & 1.2 \\
\hline Selenium & 0.3 & 0.2 \\
\hline
\end{tabular}

purchased from SSNIFF (Soest, Germany). Diet ingredient compositions of the diets are given in Tables 1-4. Throughout the study, mice were weighed once weekly. All mice were first fed HFD for 8 weeks. Four mice were immediately euthanized at the end of the eighth week of HFD (HF group). The remaining mice were switched to the CD. From these mice, 4 were euthanized after 3 days of feeding CD (3d-CD group), and the remaining 4 mice were euthanized after 1 week of feeding CD (7d-CD group). Stool pellets were collected before HFD treatment and at the end of experiment for each group.

The blood was taken via cardiac puncture while the mice were under deep anesthesia using 1\%-1.5\% isoflurane in the oxygen via anesthesia machine (EZ-SA800 Single Animal System; Elst, Plexx, Netherland) and then euthanized by cervical dislocation. Serum parameters were measured in an automatic chemistry analyzer (Hitachi, Roche, Vienna, Austria). Livers were immediately removed and cut into pieces which were (1) snap frozen in methylbutane precooled with liquid nitrogen for staining, (2) fixed in $10 \%$ buffered formalin for histological/immunohistochemical analysis, or (3) placed in liquid nitrogen for triglyceride (TG) measurement. Stool pellets were taken individually from each mouse and stored immediately at $-80^{\circ} \mathrm{C}$ until used. Animal experiments were approved by the federal animal care committee and conducted in compliance with the Austrian Law for Welfare of Laboratory Animals. (license no: BMWF-66.010/0017-II/3b/2014).

\subsection{Liver histopathology}

\subsubsection{Hematoxylin and eosin staining}

Hematoxylin and eosin (H\&E) staining was performed on 5- $\mu \mathrm{m}-$ thick formalin-fixed paraffin-embedded liver sections. Paraffin 
was removed from formalin-fixed paraffin-embedded liver sections by incubating at $65^{\circ} \mathrm{C}$ for 30 minutes followed by incubation in xylene for 15 minutes. The slides were rehydrated for 2 minutes each with decreasing concentrations of ethanol (90\%, $70 \%$, and $50 \%$ ). After a brief wash with dd $\mathrm{H}_{2} \mathrm{O}$, the liver sections were stained with hematoxylin for 2 minutes followed by 30 -second staining with eosin. Thereafter, the liver sections were washed with warm tap water for 2 minutes and mounted with Entellan (Merck, Guyancourt, France).

\subsubsection{Liver scoring}

The liver sections were analyzed on 5 fields (objective magnification 20x) per section for 3 criteria-steatosis, inflammation, and swelling of the cells-using the Brunt scoring [32]. Hepatic steatosis was assessed with a score ranging from 0 to 3 according to the accumulation of lipids in hepatocytes. A score of 0 was assigned to sections where lipids represented less than $5 \%$ of the cell, and a score of 3 was assigned to those where lipid accumulation occupied more than $66 \%$ of the cell surface. The inflammation of the tissue was also assessed with a score ranging from 0 to 3 . These ratings were attributed according to the number of inflammatory foci observed on a field. If there was none, the score was 0 ; when there were more than 4 , the score was 3 . Finally, a score between 0 and 2 was assigned according to the ballooning of the cells. When the cell had a normal size for a hepatocyte, the score was 0 ; if the shape changed and showed ballooning, particularly, if ballooning were repeated within the same field, then the score was 3 [32].

\subsubsection{Hepatic TG content}

About $50 \mathrm{mg}$ of frozen liver was homogenized in chloroformmethanol (2:1) to extract total lipids as previously described [33]. The organic extract was dried and reconstituted in isopropanol. Triglycerides were quantified using Triglycerides Colorimetric Assay kit (10010303; Cayman Chemical Company, Ann Arbor, MI, USA) and expressed as $\mathrm{mg} / \mathrm{g}$ liver.

\subsection{Microbiome analysis}

\subsubsection{DNA isolation and polymerase chain reaction amplification}

Bacterial DNA was extracted using Maxwell RSC Blood DNA Kit (Promega, Mannheim, Germany) according to the manufacturer's instructions with minor modifications. Stool samples were homogenized on a MagNA Lyser Instrument using the Lysis Buffer and MagNA Lyser Green Beads (Roche Diagnostics $\mathrm{GmbH}$, Mannheim, Germany). After homogenization, $2.5 \mathrm{mg} / \mathrm{mL}$ Lysozyme (Roth $\mathrm{GmbH}$, Karlsruhe, Germany) was added to the samples and kept for 30 minutes at $37^{\circ} \mathrm{C}$ followed by digestion with $1 \mathrm{mg} / \mathrm{mL}$ Proteinase $\mathrm{K}$ for 60 minutes at $56^{\circ} \mathrm{C}$. Enzyme activity was inactivated for 10 minutes at $95^{\circ} \mathrm{C}$, and $600 \mu \mathrm{L}$ of lysate was used for the DNA isolation in the Maxwell RSC. DNA concentration was measured using Picogreen fluorescence. The hypervariable V4 region of the bacterial 16S rRNA gene was amplified with polymerase chain reaction (PCR) from 20 ng DNA using oligonucleotide primers 16s_515_S3_fwd: GATTGCCAGCAGCCGCGGTAA and 16s_806_S2_rev: GGACTACCAGGGTATCTAAT. This 16S rDNA region was selected because of its high domain specificity and broad coverage of gastrointestinal bacteria compared with other variable regions [34]. Bacterial 16S rRNA was amplified using
Mastermix 16s Complete PCR Kit (Molzym, Bremen, Germany). The first PCR product was exposed to a second PCR with primers fusing the $16 \mathrm{~s}$ primer sequence to the $\mathrm{A}$ and $\mathrm{P}$ adapters required for Ion Torrent sequencing while additionally including a molecular barcode sequence to allow multiplexing of up to 96 samples at the same time. PCR products were separated by electrophoresis in agarose gel, and the band of the expected length (350 nt) was removed from the gel and purified using the QiaQick (Qiagen, Hilden, Germany) gel extraction system. Then, DNA concentration of the final PCR product was measured by Picogreen fluorescence.

\subsubsection{Sequencing}

Amplicons were pooled equimolarly and amplified with emulsion PCR using the Ion Torrent One Touch 2.0 Kit according to manufacturer's instructions. Then, the beads were purified on Ion ES station transferred to Ion Torrent 318 chips for sequencing. Sequencing was performed on Ion Torrent PGM using the Ion 400BP Sequencing Kit running for 1000 flows (all reagents from Thermo Fisher Scientific, Waltham, MA, USA). Sequences were sorted by barcode and transferred to the Torrent Suite server. Unmapped bam files were then used as input for bioinformatic analysis.

\subsubsection{Bioinformatics and phylogenic analysis}

All sequences were trimmed by a sliding window quality filter with a width of $20 \mathrm{nt}$ and a cutoff of Q20. Reads mapping to the human genome and reads shorter than 100 nucleotides were removed using deconseq [35]. The resulting reads were subjected to error correction using the Acacia tool [36], leading to error correction of $10 \%-20 \%$ of reads. Then, PCR chimeras were removed by usearch algorithm in de novo and referencebased settings [37]. The final sequence files were analyzed by QIIME 1.8 workflow scripts [38]. OTU search was performed using the parallel_pick_open_reference_otus workflow script and the green genes 13_8 reference database.

\subsection{Statistical analyses}

Using the QIIME core microbiome script, OTUs were visualized as OTU tables, bar charts, and PCOA plots. Additionally, groupings supplied in the mapping file were tested for statistical significance using the QIIME implementation of the Adonis test. Significance of individual bacterial strains was determined by Kruskal-Wallis test. Multiple testing corrections (false discovery rate) were used to avoid false positives (significance threshold $=0.05$ ). For statistical analysis, R 3.5 software using the package Bioconductor 3.8 and GraphPad Prism 6 were used.

\section{Results}

To investigate the effects of HFD on microbiome and liver phenotype, the following experimental setup was used: twelve 8-week-old male C57BL/6 J mice were fed HFD for 8 weeks. Four mice were then euthanized, and livers were isolated (termed as HF). The remaining mice were switched to $C D$ and euthanized after $3(n=4)$ and 7 days $(n=4)$ (termed as $3 d-C D$ and $7 d-C D$, respectively) (Fig. $1 A)$. The mice $(n=12)$ 


\section{Experiment design}

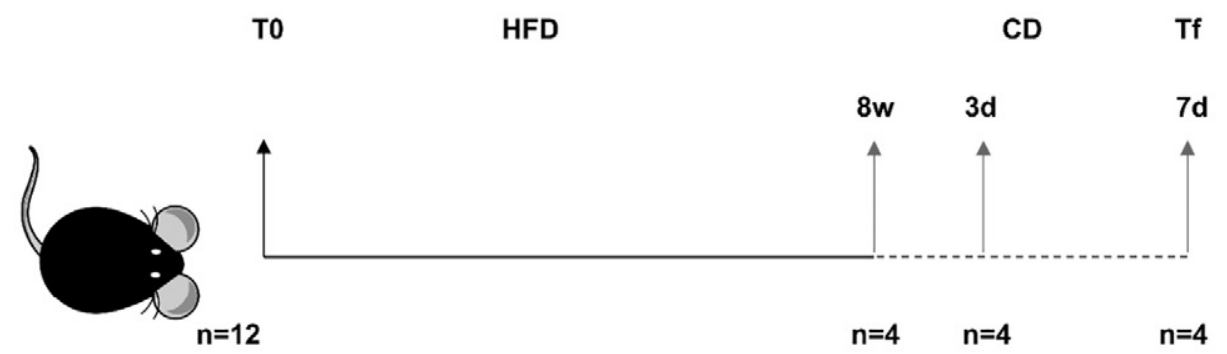

(a)

\section{Body weight}

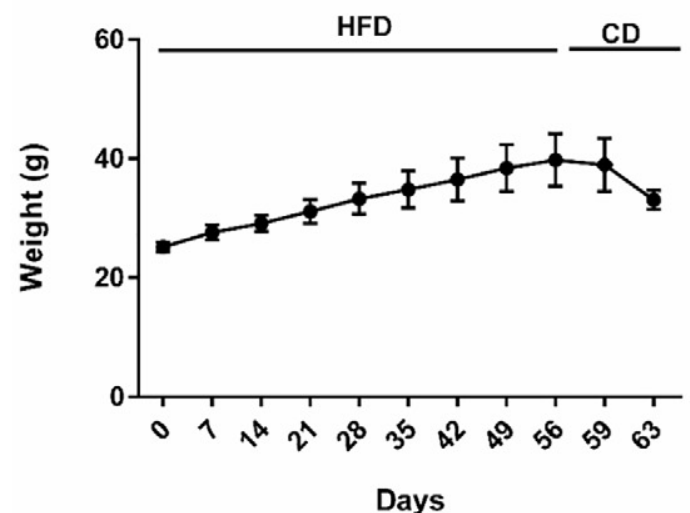

(b)

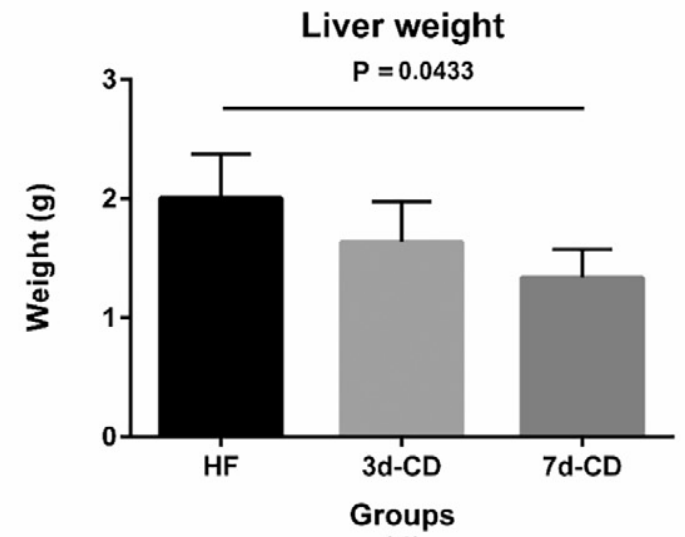

(d)

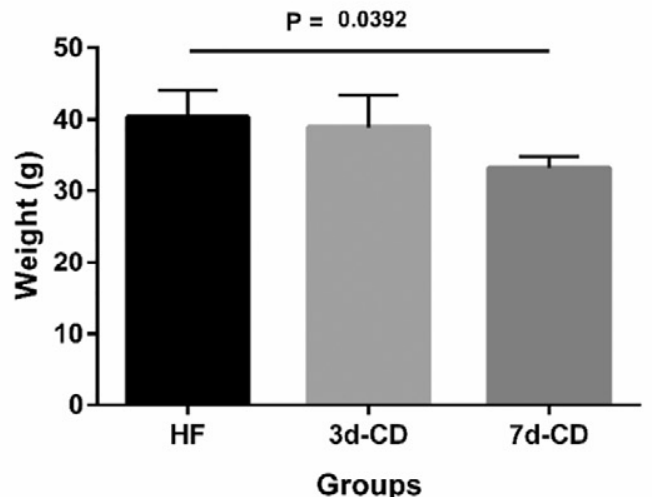

(c)

Liver/Body weight

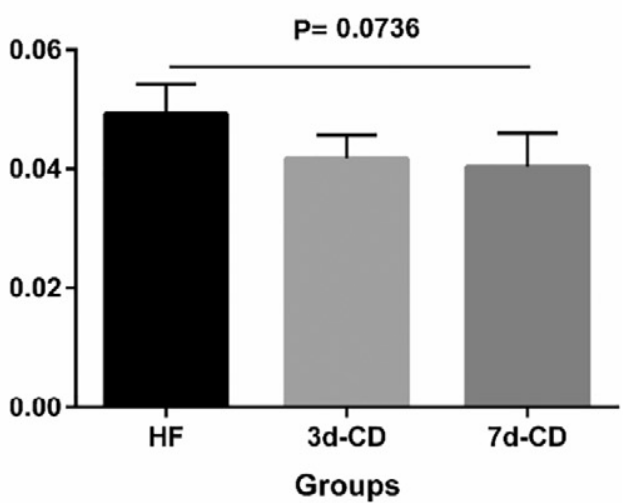

(e)

Fig. 1 - Experimental design and body and liver weights. (A) 12 mice received HFD for 8 weeks. Four mice were euthanized at the end of the HFD period (HF). The remaining mice were switched to a CD for additional 3 (3d-CD) and 7 days (7d-CD), respectively. (B) Body weight curve, (C) final body weight, (D) liver weight, and (E) liver to body weight ratio in the 3 studied groups. Values are means with error bars using SD. Groups were compared using Tukey multiple comparison test. $P$ values were calculated at a $95 \%$ confidence level. Each group contains $n=4$ mice.

displayed significant weight gain during the 8-week HFD period (Fig. 1B). The average of the weight gain for all the mice after 8 weeks of HFD was $14.43 \mathrm{~g}$. Switching to CD led to an average decrease in body weight of $1.57 \mathrm{~g}$ at 3 days and $3.03 \mathrm{~g}$ at 7 days, the latter being significant $(P=.0392)$ (Fig. $1 C)$. The liver weight in the 7d-CD group also showed significantly lower value compared to the HF group ( $P=.0433$ ) (Fig. 1D). Moreover, the liver to body ratio in the same group also showed a trend to be lower as compared to the HF group $(P=.0736)$ (Fig. 1E). 


\section{1. $C D$ reverses the HFD-induced steatosis}

Histology and liver scoring showed evidence of steatosis in the HF and 3d-CD groups, whereas steatosis was absent in the $7 d-C D$ group. No inflammation and ballooning were observed in the liver of any mouse (Fig. 2A and C). The mice with 1 week of CD showed significantly lower fat content in the hepatocytes, indicating that 7 days of $C D$ is necessary and sufficient to reverse 8-week-HFD-induced steatosis in C57BL/6 J mice (Fig. 2A). The triglyceride assay also confirmed the significant difference in the fat deposited in the liver between the HF group (7.237 ng/mg tissue) and the group additionally treated for 1 week with CD (7dCD) $(2.990 \mathrm{ng} / \mathrm{mg}$ tissue) $(P=.0132)$ (Fig. 2B). A significant difference in the TG levels was also revealed between the $3 \mathrm{~d}-\mathrm{CD}$ (6.457 ng/mg tissue) group and 7d-CD group ( $\mathrm{P}=$ .0372), further supporting that 7 days of $C D$ is needed to reverse hepatic triglyceride accumulation (Fig. 2B).

Based on these results, we chose to compare $7 d-C D$ with $\mathrm{HF}$ to further investigate the capacity of $\mathrm{CD}$ to reverse the effects of HFD feeding. Therefore, the following figures will present results obtained in mice euthanized after 8 weeks of HFD (HF group) and in mice euthanized after 8 weeks of HFD followed by 7 days of CD (7d-CD group).
To better define the ability of 7 days of $C D$ to reverse the adverse metabolic effects of HFD, we assayed serum parameters including albumin, alkaline phosphatase, triglyceride, lactate dehydrogenase, aspartate aminotransferase, alanine transaminase, glucose, and cholesterol. Among there parameters, glucose and cholesterol were found to be significantly reduced in 7d-CD vs HF group $(P<.0001$ and $P=.0216$, respectively) (Table 5). In addition, the ALT level showed a trend for being decreased in $7 d-C D$ vs HF group $(P=.154)$. No differences were found between the 2 groups for the other parameters measured (Table 5).

\subsection{CD reverses the HFD-induced dysbiosis}

Replacing HFD with CD for 1 week normalized body weight and reversed the hepatic steatosis observed in HF group mice. To decipher if these changes in mice phenotype were associated with modifications of the microbiome, the stool pellets from mice of both groups (HF and 7d-CD) were taken before HFD treatment (TO) and at the end of experiment for each group (after 8 weeks of HFD for HF group [HF-Tf] and after 8 weeks of HFD followed by 1 week of CD for 7d-CD group [7d-CD-Tf]) and analyzed using 16S rRNA sequencing.
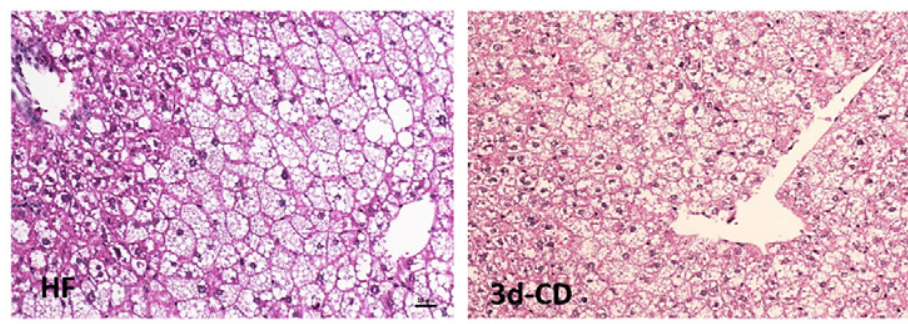

(a)

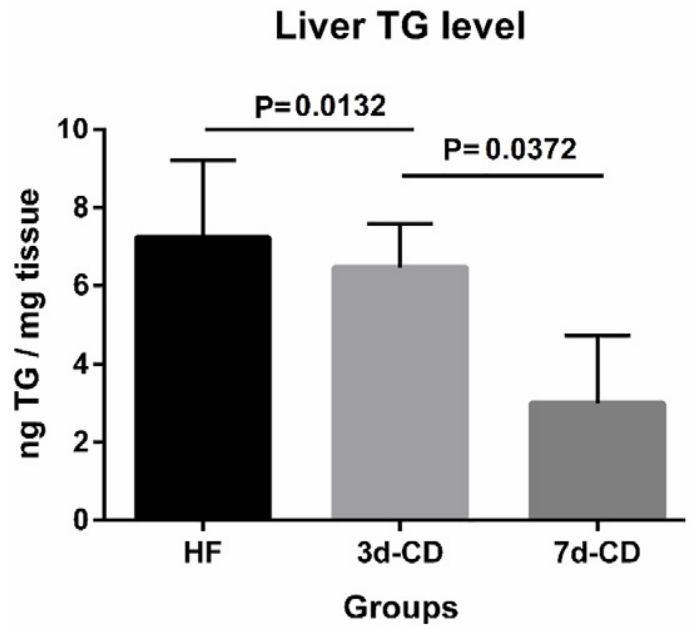

(b)

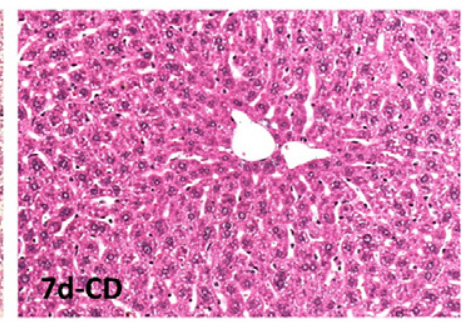

(7d-CD 1) M

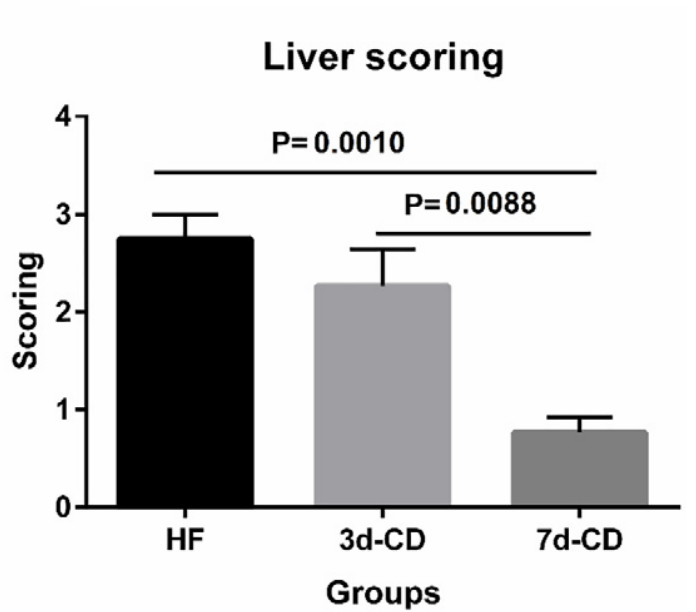

(c)

Fig. 2 - (A) Representative H\&E staining of liver sections (5 $\mu \mathrm{m}, 20 \times$ magnification). The histology of the liver stained with H\&E technique showed that the liver of the mice treated with control diet for 7 days after 8 weeks of HFD treatment (7d-CD) had less fat and displayed a normal status compared to HF (fed with HFD) and 3d-CD (fed with HFD plus 3 days of control diet) groups. (B) Quantification of liver TG levels per tissue weight (mg) in HF, 3d-CD, and 7d-CD groups. (C) Liver scoring. Histological grading and staging system for liver steatosis was based on the Brunt scoring described by Tiniakos et al [32]. Values are means with error bars using SD. Groups were compared using Tukey multiple comparison test. P values were calculated at a $95 \%$ confidence level. Each group contains $n=4$ mice. 
Table 5 - Serum parameters measured in HF (fed with HFD for 8 weeks) vs 7d-CD (fed with HFD for 8 weeks plus 1 week of control diet) groups

\begin{tabular}{|c|c|c|c|c|c|c|}
\hline \multirow[b]{2}{*}{ Groups } & \multicolumn{3}{|c|}{ Alanine aminotransferase (U/L) } & \multicolumn{3}{|c|}{ Alkaline phosphatase (U/L) } \\
\hline & Mean & SD & $P$ value & Mean & SD & $P$ value \\
\hline HF & 20.5000 & 8.7369 & \multirow{2}{*}{.1548} & 22.2500 & 4.2720 & \multirow{2}{*}{.4224} \\
\hline \multirow[t]{2}{*}{$7 \mathrm{~d}-\mathrm{CD}$} & 12.5000 & 4.5092 & & 18.5000 & 7.5939 & \\
\hline & \multicolumn{3}{|c|}{ Aspartate aminotransferase (U/L) } & \multicolumn{3}{|c|}{ Serum albumin $(g / d L)$} \\
\hline Groups & Mean & SD & $P$ value & Mean & SD & $P$ value \\
\hline \multirow{3}{*}{$\begin{array}{l}\text { HF } \\
7 d-C D\end{array}$} & 70.0000 & 58.3267 & \multirow{2}{*}{.6437} & 1.1250 & 0.0500 & \multirow{2}{*}{.0871} \\
\hline & 55.0000 & 19.9332 & & 0.9250 & 0.1893 & \\
\hline & \multicolumn{3}{|c|}{ Serum cholesterol (mg/dL) } & \multicolumn{3}{|c|}{ Serum glucose $(\mathrm{mg} / \mathrm{dL})$} \\
\hline Groups & Mean & SD & $P$ value & Mean & SD & $P$ value \\
\hline \multirow{3}{*}{$\begin{array}{l}\text { HF } \\
7 d-C D\end{array}$} & 57.5000 & 1.9149 & \multirow{2}{*}{$<.0001$} & 86.0000 & 5.4772 & \multirow{2}{*}{.0216} \\
\hline & 30.5000 & 2.3805 & & 67.7500 & 10.5000 & \\
\hline & \multicolumn{3}{|c|}{ Lactate dehydrogenase (U/L) } & \multicolumn{3}{|c|}{ Serum triglyceride (mg/dL) } \\
\hline Groups & Mean & SD & $P$ value & Mean & SD & $P$ value \\
\hline $\mathrm{HF}$ & 264.7500 & 170.6778 & \multirow{2}{*}{.6119} & 23.5000 & 5.1962 & \multirow{2}{*}{.3679} \\
\hline $7 d-C D$ & 213.7500 & 84.9799 & & 20.7500 & 2.2174 & \\
\hline
\end{tabular}

Data are presented as means with standard deviation; $\mathrm{n}=4$ per group. For comparing the groups, an unpaired $t$ test was used. $\mathrm{P}$ values were calculated at a $95 \%$ confidence level.

Rarefaction curves suggested that richness was lower at the end of the experiment in both HF and 7d-CD groups compared to T0, indicating that HFD reduced the bacterial richness which was not restored by 1 week of CD (Fig. 3). Multidimensional scaling analysis first showed that before HFD treatment (T0), all mice clustered together, indicating similar gut microbiota composition (Fig. 4). HFD treatment greatly modified the microbiota composition leading to a separate cluster (HF-Tf). Finally, 7 days of CD led to a new cluster (7d-CD-Tf) closer but slightly different from the original one. These results indicate that HFD strongly affects

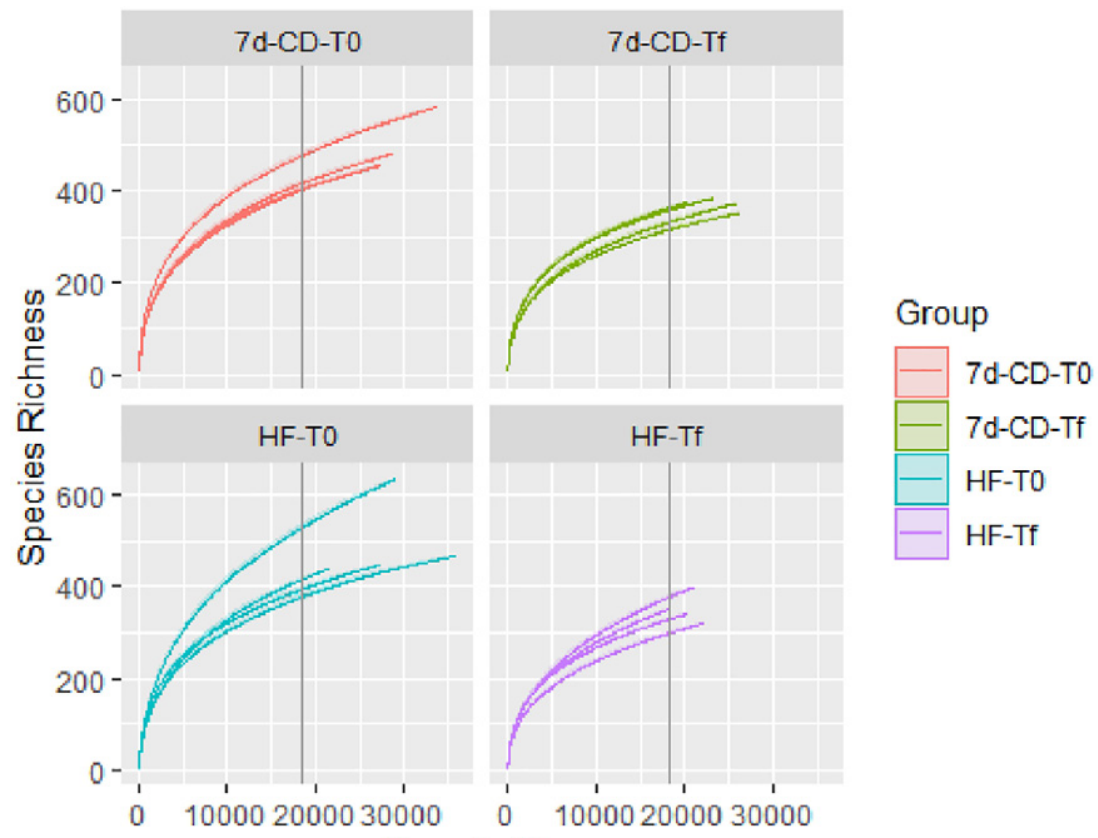

Sample Size

Fig. 3 - Rarefaction curves used to estimate richness in HF (fed with HFD for 8 weeks) vs 7d-CD (fed with HFD for 8 weeks plus 1 week of control diet) group at T0 (before HFD treatment) and Tf (after HFD or after HFD and control diet). 


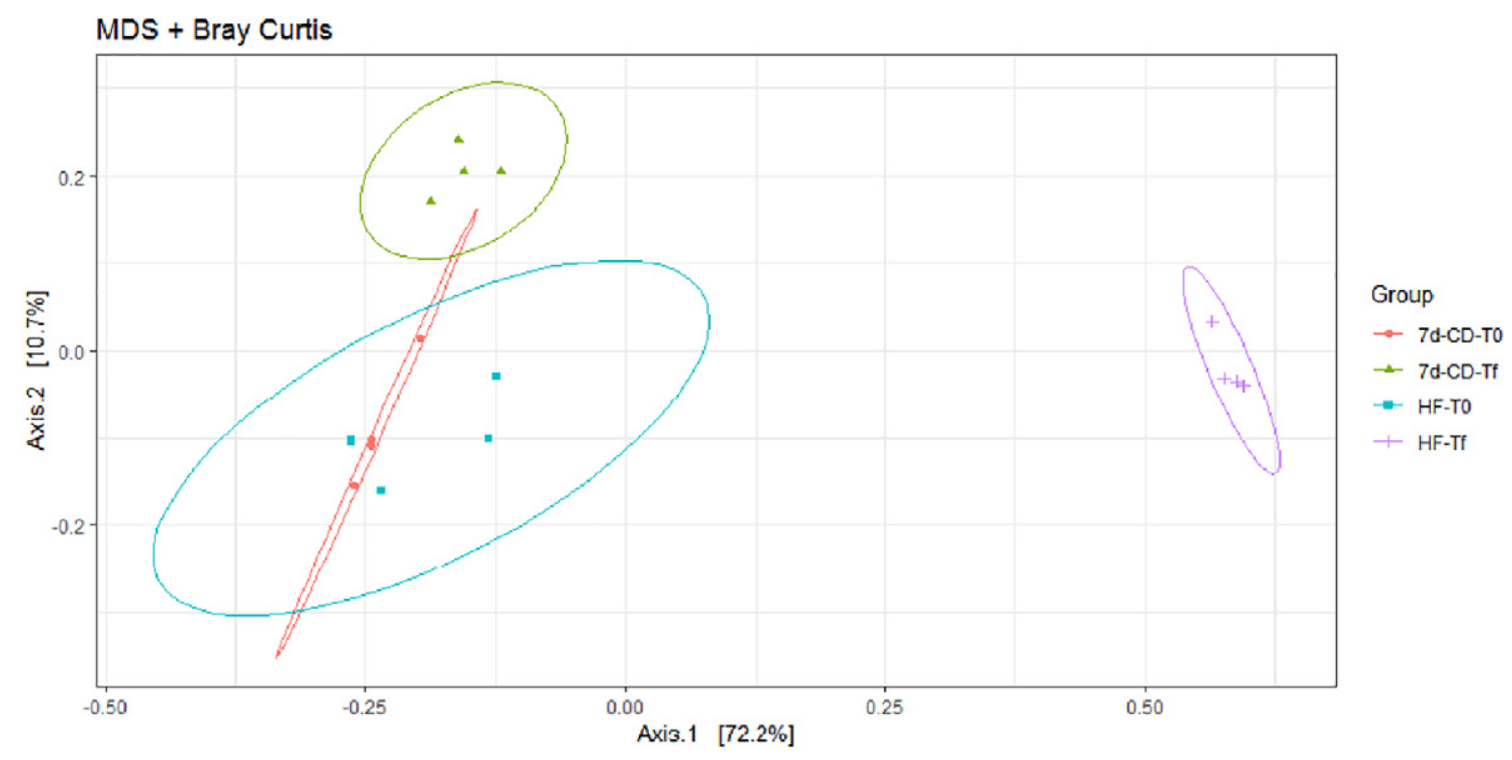

Fig. 4 - $\beta$-Diversity analysis via nonmetric multidimensional scaling of Bray-Curtis Dissimilarity Indices. Each dot represents an animal, projected onto first (horizontal axis) and second (vertical axis) variables. The ellipse determines the $95 \%$ confidence interval.

gut microbiota composition, which is partially restored by 1 week of $\mathrm{CD}$.

Using a Venn diagram, we compared the OTUs shared between by the mice before (TO) $(n=8)$ and after treatment (HF$\operatorname{Tf}[\mathrm{n}=4]$ and $7 \mathrm{~d}-\mathrm{CD}-\mathrm{Tf}[\mathrm{n}=4])$. The results confirmed $\beta$-diversity analysis which showed that bacterial community from 7d-CD-Tf is closer to initial bacterial community than the one from HF-Tf. Indeed, 268 OTUs were shared between baseline (T0) and 7d-CDTf microbiomes, whereas only 183 OTUs were shared between baseline (T0) and HF-Tf microbiomes (Fig. 5). Interestingly, 151

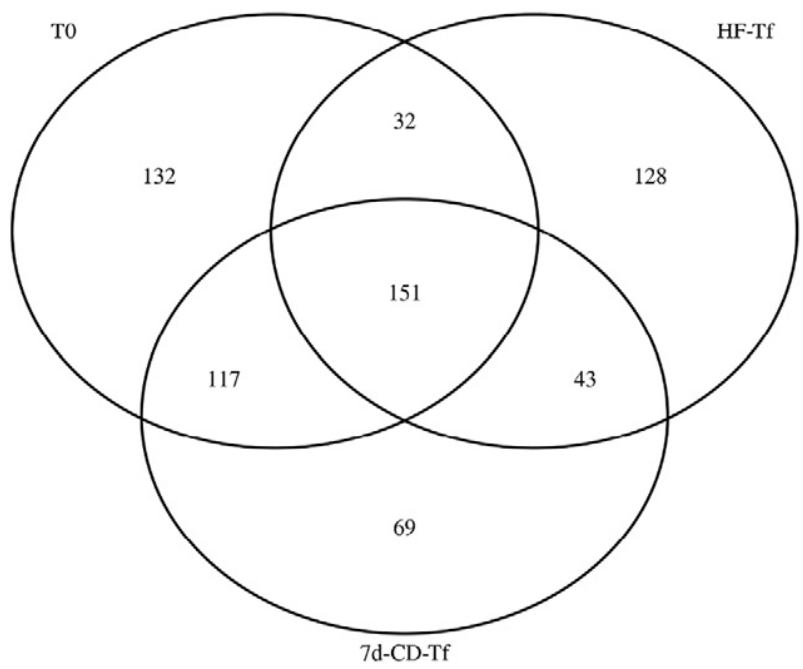

Fig. 5 - Venn diagram based on OTUs distribution (at 3\% dissimilarity) showing the OTUs shared by the mice before (T0) $(n=8)$ and after treatment (HF-Tf $[n=4]$ and 7d-CD Tf [n= 4]).
OTUs were present in the 3 compared microbiomes, indicating that the presence of these bacterial species was not affected by the changes in diet (Fig. 5).

The 16S rRNA gene sequencing of mice fecal samples revealed that the observed species and the richness (Chao index) were reduced in both HF and 7d-CD groups, further indicating that diversity was decreased by HFD and not restored by 1 week of CD (Fig. 6). However, Shannon diversity index was found higher in 7d-CD-Hf compared to HF-Tf, indicating a less biased community structure (more equal abundances of the bacterial species) after 7 days of CD (Fig. 6).

To further assess the effects of the diet treatments on microbiota, we analyzed microbiota composition in mice before (HF-T0) and after (HF-Tf) HFD, and before (7d-CD-T0) and after (7d-CD-Tf) HFD + 1 week of CD. In all mice groups, the most abundant phyla include Bacteroidetes, Deferribacteres, Firmicutes, Tenericutes, and Verrucomicrobia (Fig. 7). The population of Bacteroidetes was decreased by the HFD treatment, whereas it was found to be increased in $7 d-C D$ mice at the end of the experiment (Figs. 7 and 8). HFD treatment also led to increased populations of Firmicutes and Actinobacteria which were not found in 7d-CD mice. Accordingly, the Firmicutes to Bacteroidetes ratio was found to be significantly higher in HF-Tf group compared to other studied groups. Tenericutes almost disappeared following HFD treatment, whereas its abundance was similar in 7d-CD group before and after treatment (Figs. 7 and 8). These results indicate that, at the phylum level, 1 week of CD is sufficient to markedly abolish HFD-induced microbiota changes.

At the family level, Porphyromonadaceae, Clostridiaceae, Lactobacillaceae, and Erysipelotrichaceae were highly increased by the HFD treatment, whereas their abundance was similar in 7d-CD group before and after treatment (Figs. 9 and 10), indicating that 1 week of CD is sufficient to abolish the impact of 8 weeks of HFD on these bacterial populations. 

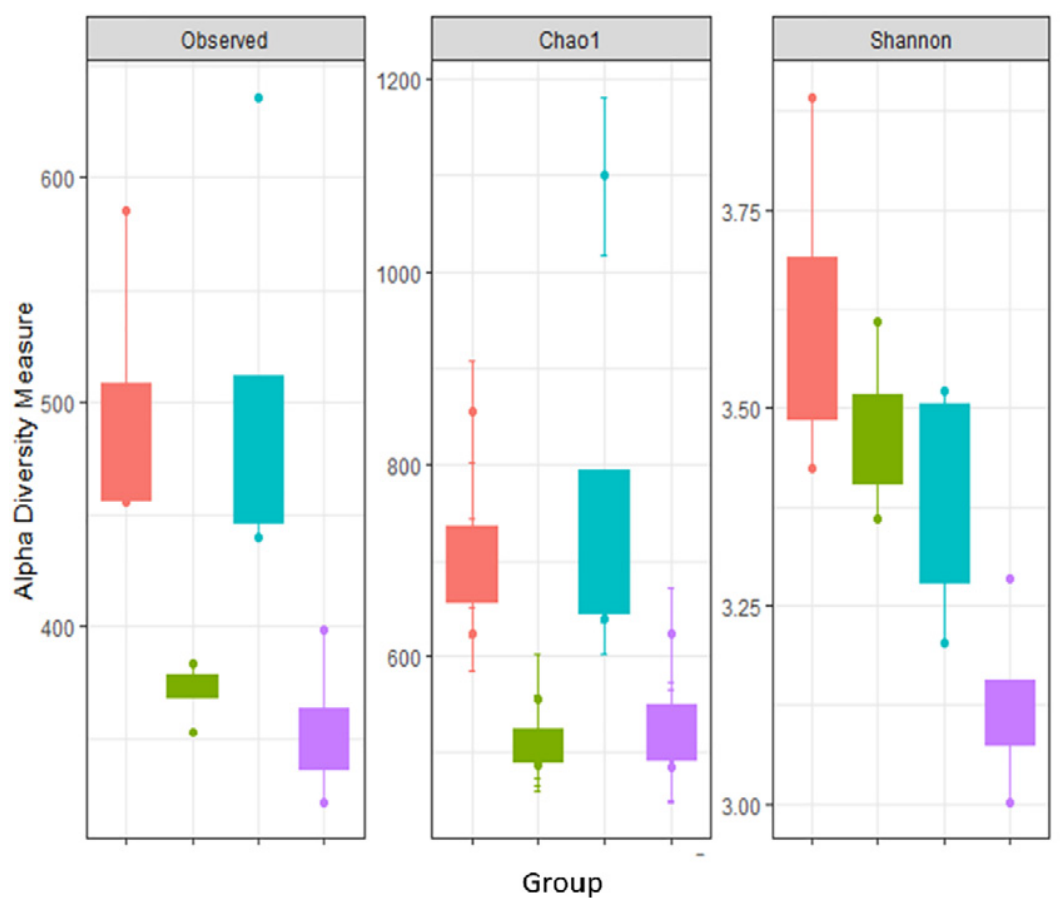

Group

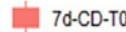

7d-CD-If

HF-TO

HF-Tf

Fig. 6 - $\alpha$-Diversity indices in fecal samples of mice before and after treatment in 7d-CD and HF groups. Chao1 estimates the number of species, whereas Shannon estimates the effective number of species. Each group contains $n=4$ mice.

Similarly, Prevotellaceae disappeared after HFD treatment, whereas 1 week of CD restored its population $(P<.0001)$. Conversely, Anaeroplasmataceae populations disappeared following HFD treatment, which was only slightly corrected by 1 week of $C D$, indicating an uncomplete restoration of the baseline microbiota (Fig. 10).

At the genus level, Parabacteroides, Allobaculum, Lactobacillus, and an unknown genus in the Erysipelotrichacease family were

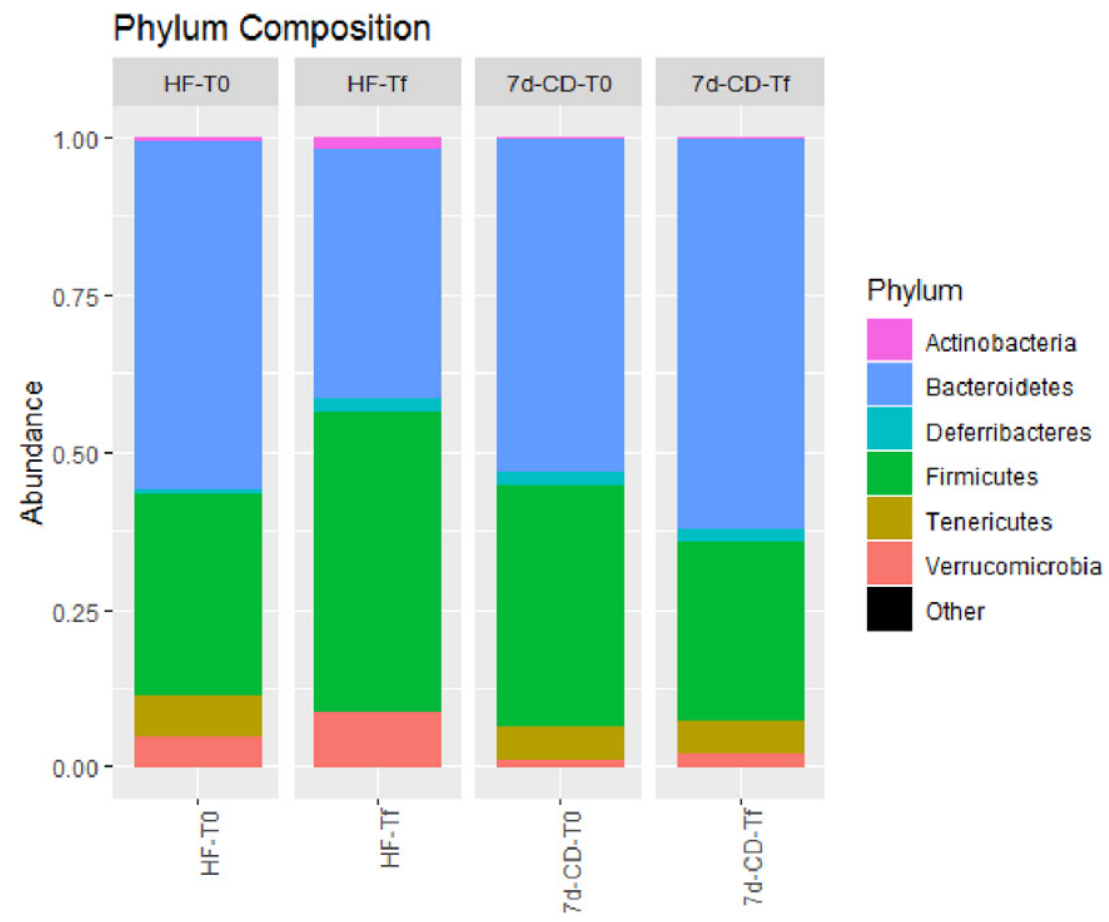

Fig. 7 - Microbiota composition at the phylum level (relative abundance) in HF and 7d-CD groups at T0 and Tf. Each group contains $n=4$ mice. 

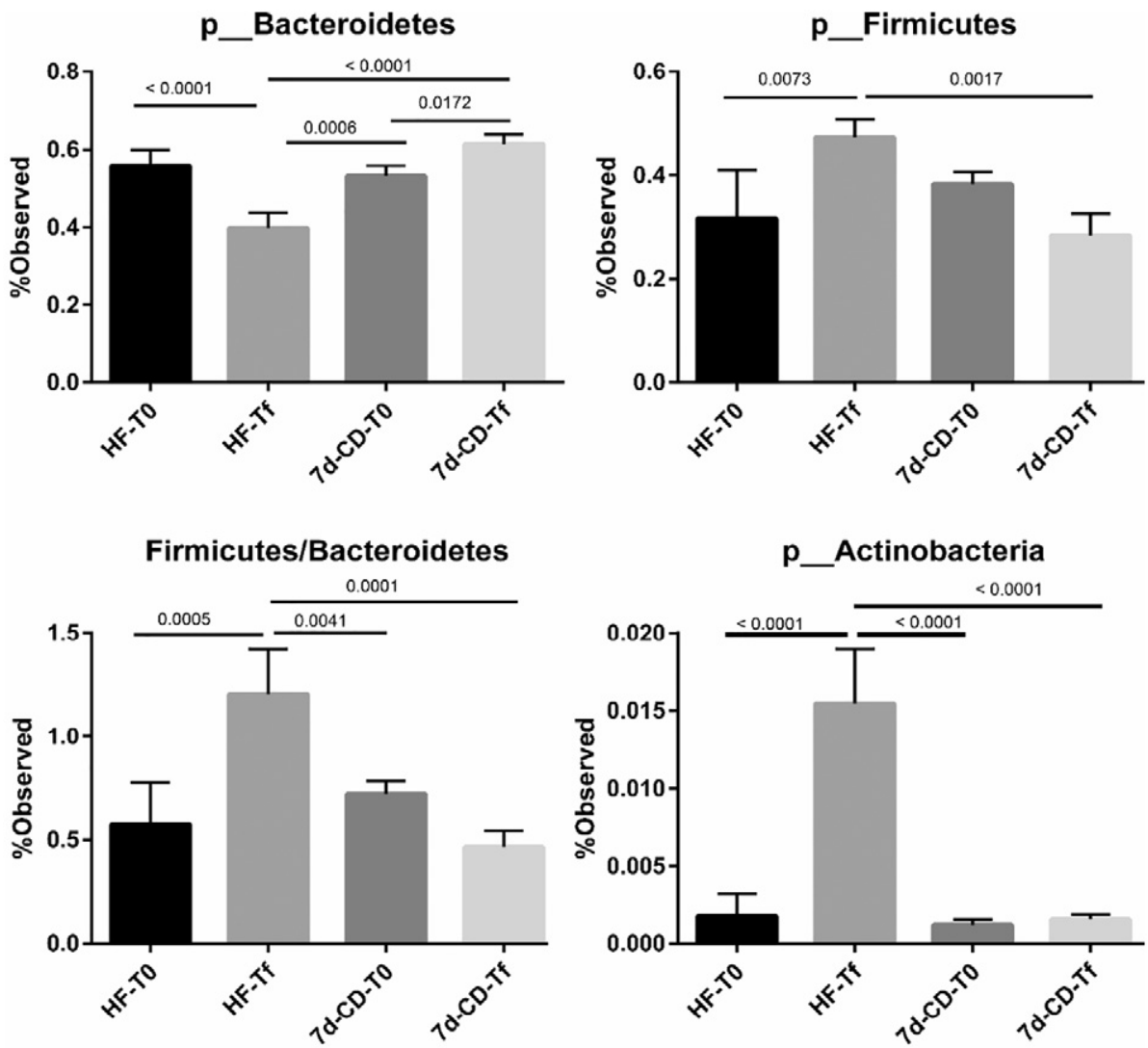

\section{p_Tenericutes}

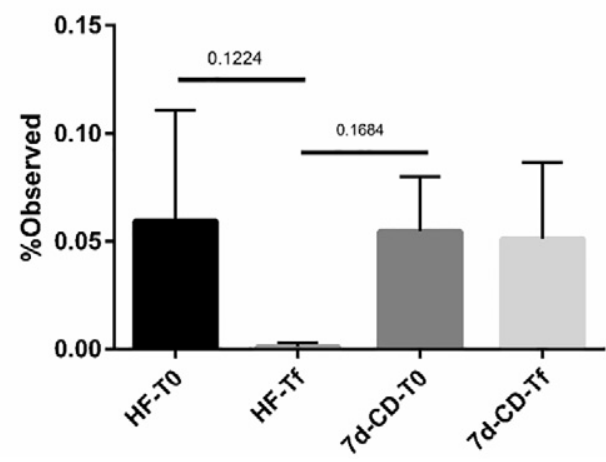

Fig. 8 - Impact of diet treatments on the relative abundances of phyla. Groups were compared using Tukey multiple comparison test. $P$ values were calculated at a $95 \%$ confidence level. Each group contains $n=4$ mice.

boosted by HFD treatment. These increases were not found in 7dCD-Tf, suggesting that 1 week of CD completely abolished this effect (Fig. 11). HFD also caused the eradication of Prevotella and Anaeroplasma, and the CD led to restoration of Prevotella only, further indicating that 1 week of $\mathrm{CD}$ led to a marked but uncomplete restoration of the baseline microbiota.

\section{Discussion}

In the experimental setup used in this study, mice were fed HFD for 8 weeks followed by a change to CD to first describe how HFD affects body weight gain, liver phenotype, and gut microbiota composition in mice and then to evaluate the reversibility of these HFD-induced effects. We first evaluated the effects of HFD on host parameters and found that 8 weeks of HFD induced obesity and steatosis in mice. In line with our results, Zeng et al [31] studied C57BL/ 6 mice fed an HF (45\% energy) or low-fat ( $10 \%$ energy) diet for 10 weeks. The HFD-fed mice increased their body weight by $34 \%$ compared to the low-fat-fed mice, and the liver of these mice also exhibited a dramatic increase in the number of lipid droplets. Then we aimed to investigate if these HF-induced metabolic disorders are reversible after switching HF to CD. In our study, 3 days of CD after 8 weeks of HFD treatment was not able to reverse the fatty liver phenotype and weight gain due to HFD treatment. Conversely, the liver recovers and shows essentially normal phenotype after 7 days of CD. One week of CD following 8 weeks of HFD led to reduced 


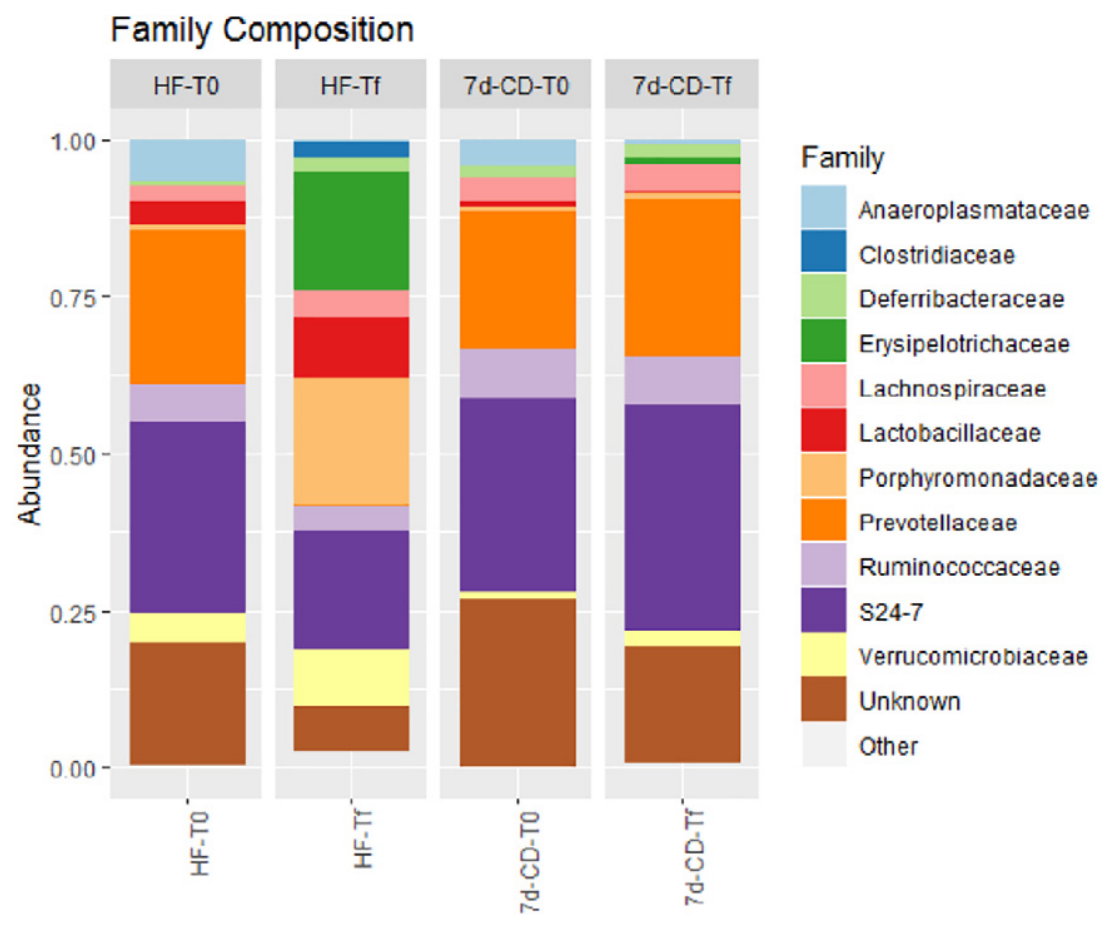

Fig. 9 - Microbiota composition at the family level (relative abundance) in HF and 7d-CD groups at T0 and Tf. Each group contains $n=4$ mice.

amount of fat deposition in hepatocytes which was also confirmed by TG assay and scoring. Kowalski et al [27] used HFD (42\% energy from fat and $20 \%$ from sucrose) for 56 days followed by 9 days of ad libitum CD. Glucose intolerance and hyperinsulinemia were completely normalized, whereas fat mass and liver triglycerides showed an intermediate phenotype. The diet reversal also resulted in significant reductions in hepatic de novo lipogenesis when compared to the high-fat, high-sucrose diet groups. Altogether, these results and ours indicate that the metabolic and hepatic disorders induced by HFD are mainly reversible with a minimum of 1 week of CD being necessary to recover close to initial levels.

Together with effects on host metabolic parameters, HFD is responsible for rapid changes in microbiome composition. Indeed, it was observed that switching from a low-fat, plant polysaccharide-rich diet to a high-fat, high-sugar Western diet shifted the structure of the microbiota within a single day, changed the representation of metabolic pathways in the microbiome, and altered microbiome gene expression [22]. However, the shifts in response to daily variation in the diet are shown to be at the genus and species level rather than at the phylum level, and long-term diet has been proven to be the primary driver in determining one's gut microbiota [39]. Moreover, different types of high-fat diet, with different percentages of saturated and polyunsaturated fatty acids, were shown to have different effects on the gut microbiota [40]. Carmody et al [41] studied the effect of dietary perturbations on the gut microbiota of 5 inbred mouse strains, showing that consumption of a high-fat, high-sugar diet reproducibly changed the gut microbiota in spite of the differences in host genotype. Particularly, the gut microbiota displayed a linear dose-dependent response to dietary perturbations, and each diet-responsive bacterial group took an average of 3.5 days to reach a new steady state [41]. In our study, the gut microbiota community of the HF group at 8 weeks clustered separately from that of initial microbiome before HF feeding, indicating a broad effect of HF on gut microbiota composition. Moreover, both diversity and richness of the microbiome were decreased by the HFD treatment in accordance with previous studies [22,41].

Human populations with a modern Western diet or a rural diet showed distinct gut microbiota structures [42], and we may speculate that these changes in gut microbiota could contribute to the higher incidence of metabolic diseases including obesity and NAFLD occurring in Western populations. Therefore, it is of primary importance to assess the capability of a normal CD to restore a balanced gut microbiota that can contribute to acquiring a healthy status. The diversity of bacteria in HF group is decreased in our study as previously found by others. A decreased diversity of the microbiota has also been observed in obese individuals, where it is correlated to the reduction in insulin sensitivity and the stimulation of inflammation [43,44]. Regarding liver diseases, alterations such as lower diversity and a phylum-level change in the fecal microbiome has been demonstrated in NAFLD $[45,46]$. Thus, our finding of a general dysbiosis characterized by a decreased diversity in mice administered an HFD for 8 weeks raises the possibility that this imbalanced microbial ecology plays a role in increasing the susceptibility to liver diseases. This is in line with other studies showing that the changes in gut microbiota induced by HFD vs CD may act as an important mediator in the etiology of obesity and related metabolic diseases, probably via disrupting regulation of lipid metabolism and inducing low-grade inflammation [47-49]. 


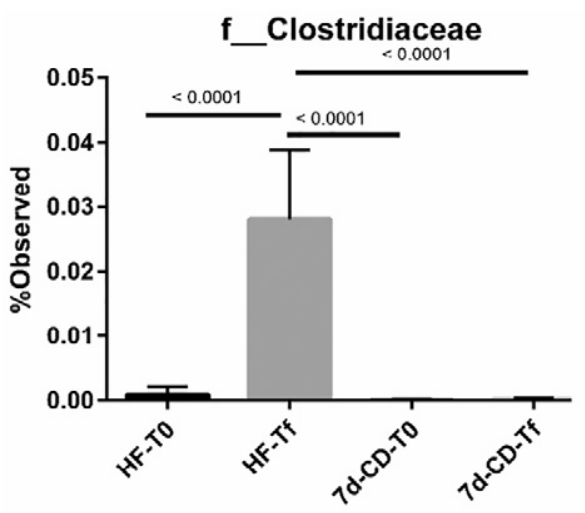

f_Erysipelotrichaceae

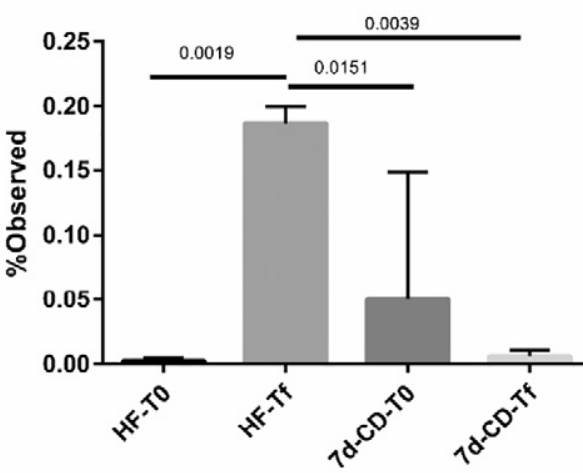

f_Prevotellaceae

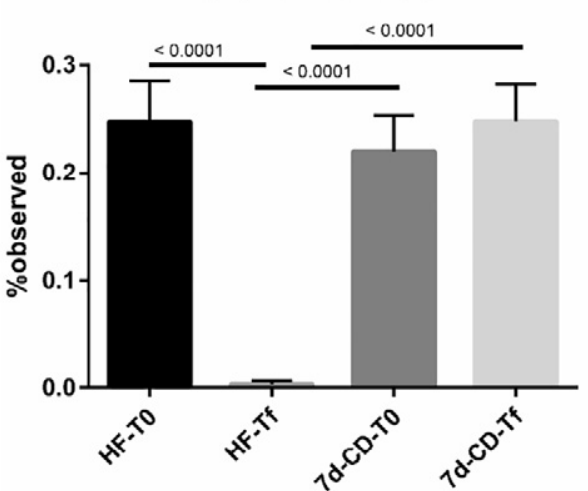

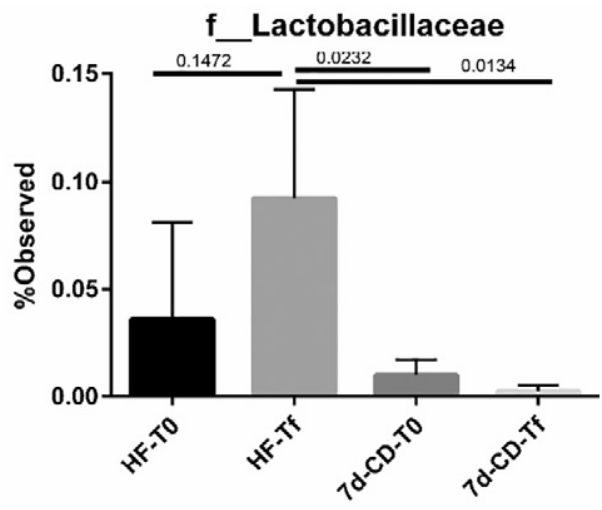

f__Porphyromonadaceae

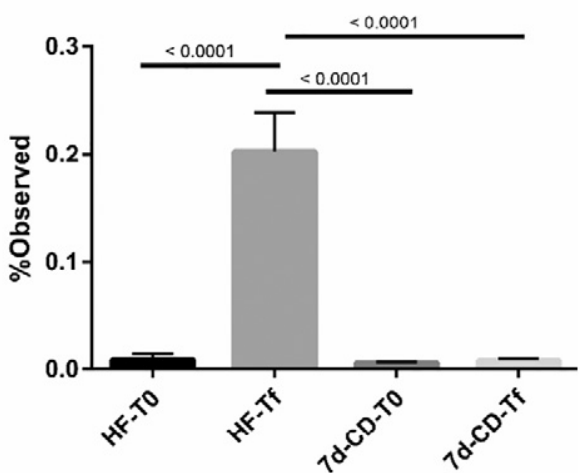

f__Anaeroplasmataceae

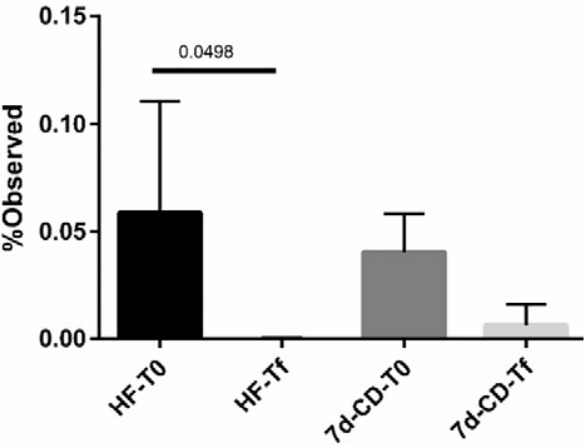

Fig. 10 - Impact of diet treatments on the relative abundances of bacterial families. Groups were compared using Tukey multiple comparison test. $P$ values were calculated at a $95 \%$ confidence level. Each group contains $n=4$ mice.

In our study, the abundance of Actinobacteria, Firmicutes, Clostridiaceae, Erysipelotrichaceae, Porphyromonadaceae, Parabacteroides, and Allobaculum increased significantly after HFD treatment for 8 weeks. Concurrently, Bacteroidetes, Prevotellaceae, and Prevotella were significantly underrepresented at the end of HFD treatment. Consistent with these findings, we have previously observed increased Allobaculum (a genus in Erysipelotrichaceae) after HFD consumption [9]. Moreover, decreased Prevotella [50] and increased Allobaculum numbers in HFD-fed mice were also reported by other studies $[50,51]$. HFD feeding modulates the gut microbiota composition by decreasing the prevalence of specific gut barrierprotecting bacteria and increasing the prevalence of opportunistic pathogens that can release free antigens such as lipopolysaccharides. This imbalance may be associated with a higher gut permeability, leading to higher plasma levels of endotoxin and inducing inflammation and eventually the development of metabolic disorders, a phenomenon termed metabolic endotoxemia [48,49].

Structural resilience is the ability of a complex system to recover to its normal state after removal of the perturbation. Microbiome reversibility has been particularly studied in the context of antibiotic treatments. As an example, after disruption by antibiotics, the structure of the gut microbiota began to return to its initial state by 1 week after the end of each antibiotic course but did not reach complete recovery 

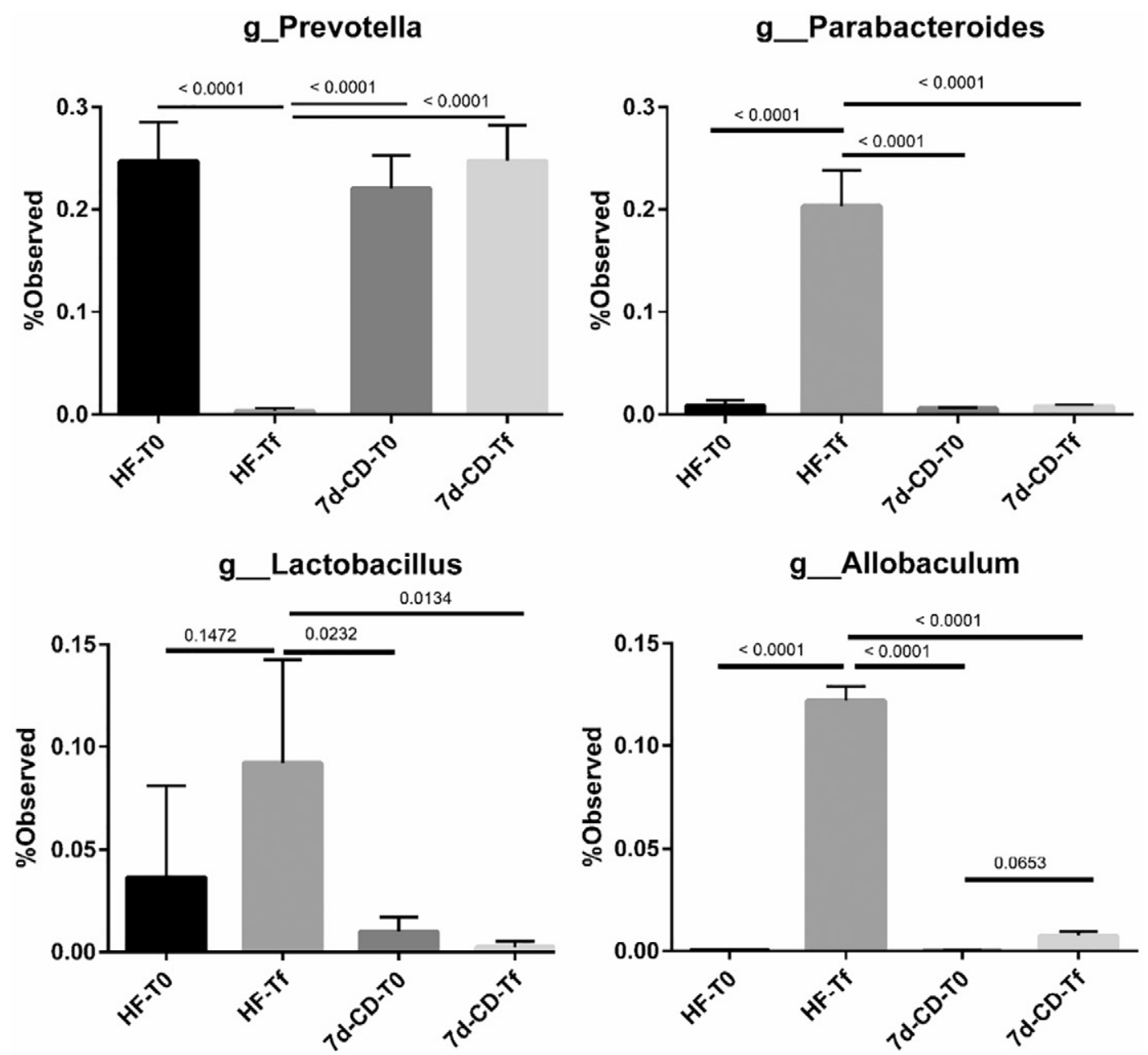

\section{f_Erysipelotrichaceae;g_}

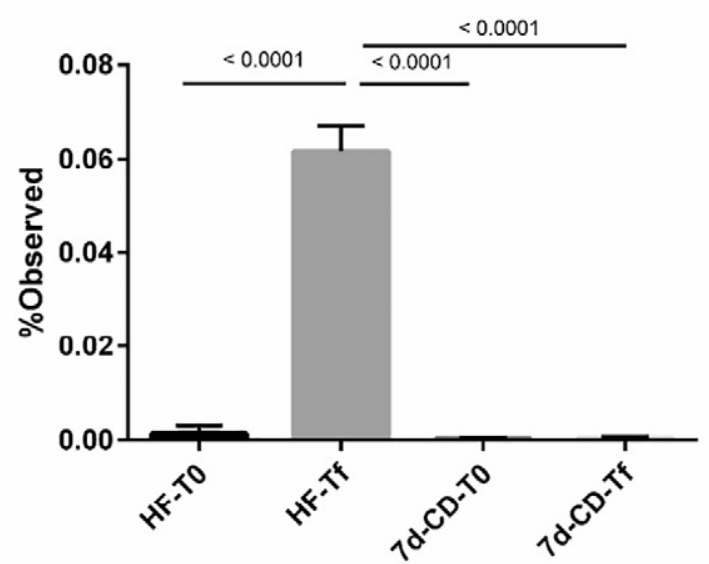

Fig. 11 - Impact of diet treatments on the relative abundances of bacterial genera. Groups were compared using Tukey multiple comparison test. $P$ values were calculated at a $95 \%$ confidence level. Each group contains $n=4$ mice.

even after 5 months [52]. However, it is not yet known to what extent the HFD-disrupted structure of the gut microbiota can be reversed after returning to a balanced diet. One of the dieting problems in the age of the obesity epidemic is the cycle of weight loss and regain known as the yo-yo effect. Thaiss et al [29] demonstrated that the microbiome plays a key role in this phenomenon, as HF-induced changes to the microbiome persist over long periods of time and enhance the rate of weight gain during secondary HF challenge. Zhang et al [51] monitored the dynamic changes of the gut microbiota in C57BL/6J mice fed with a 34.9\% fat HFD for 12 weeks and then reverted to $C D$ for 10 weeks. HFD feeding significantly 
influenced the richness and diversity of the bacterial community, which was reversible upon reverting to normal diet feeding. They observed increased number of Firmicutes and Proteobacteria and decreased number of Bacteroidetes in the group fed with HFD and their reversal after receiving normal diet. In our study, we assessed whether feeding our mice with CD for a shorter period (7 days) was able to reverse the effect of HFD ( $60 \%$ energy from fat) on the gut microbiota composition. Based on our findings, HFD caused increased frequency of Firmicutes and Firmicutes/Bacteroidetes and decreased Bacteroidetes, which are in agreement with some other studies [51,53,54]. Our findings also showed significantly higher amount of Actinobacteria in HF group vs $7 d-C D$ group, indicating that this phylum is highly susceptible to diet changes and that its HFD-induced increase is reversible upon changing diet. This confirms the results of previous studies indicating that Actinobacteria abundance is positively associated with an HFD and negatively associated with fiber intake $[40,54]$. At the family level, we observed that Erysipelotrichaceae, Lactobacillaceae, Clostridiaceae, and Porphyromonadaceae bloomed in HF group and showed a significant drop in number after treatment with CD reaching close to initial level. Zhang et al [49] identified 4 different lineages within Erysipelotrichaceae that respond differently to diet or host health phenotypes, whereas some studies observed an increase of Erysipelotrichaceae in mice on HF or Western diet $[22,50,54,55]$ in accordance with our results. In addition, our data revealed a significantly reduced level of hydrogen-producing Prevotellaceae following HF feeding and its repopulation after normal diet in 7d-CD group, which are in accordance with the findings of Heisel et al [50]. In contrast, Zhang et al [56] showed obesity to be associated with an increase of family Prevotellaceae prior to the surgery, and following surgery, Prevotellaceae was reduced to the level of lean individuals. Also, human studies have shown higher levels of Prevotellaceae in obese individuals compared with lean subjects and with those after gastric bypass [57,58]. Indeed, obesity is not systemically due to a lipid-rich diet, and gut microbiota composition in obese individuals results from many factors and does not always correlate with the effects of HF observed in animal models. This further suggests that this bacterial family does not play a role in metabolic phenotypes, as it has been found increased or decreased in humans and animals with obesity or steatosis. At the genus level, Allobaculum and Parabacteroides were also overrepresented in HF group and remarkably reduced after 7 days of treatment with the CD. Conversely, Prevotella showed a reduced frequency in HF-fed mice, and its amount was elevated in mice treated with CD. We also observed an increased number of Lactobacillus in HF group, which was reduced after CD. Lactobacillus may be associated with the production of volatile organic compounds such as acetate and ethanol [59], which may be involved in the pathogenesis of obesity and NAFLD. In human studies, a significantly higher level of Lactobacillus species (from the phylum Firmicutes) was also found in obese patients than in lean controls [60].

These results revealed that HF feeding highly impacts gut microbiome composition but that 7 days of CD is sufficient to reverse the majority of HF-induced microbiota changes. Because metabolic parameters were reversible in parallel, we may postulate that gut microbiota changes contribute to the changes in liver and metabolic phenotype. Using gut microbiota transplant experiments, we have already shown that gut microbiota composition determines steatosis development in recipient mice [9]. Transplantation of microbiota taken after HFD treatment and after CD restoration could be used in the future to decipher if the associated microbiota changes are responsible for improvement of steatosis. The intestinal microbiota has been extensively studied at both the phylogenetic and metagenomic levels in the context of metabolic disorders $[41,61,62]$. The novelty of the present work lies in the investigation of the reversibility of HFDinduced dysbiosis and its association with development and improvement of the fatty liver disease. Among the bacterial groups that were found to change in parallel with steatosis in our study, the family Erysipelotrichaceae is emerging as a group of bacteria that may influence host metabolism and inflammatory diseases [63,64]. Interestingly, Spencer et al [30] showed a positive association of the Erysipelotrichi abundance with the changes in liver fat in women who were placed on diets in which choline levels were manipulated. We also found that Lactobacillus was associated with fatty liver and was found reversible upon diet changes. Accordingly, a positive correlation between the amount of Lactobacillus DNA in the fecal samples and the severity of steatosis within mouse livers was already observed. The authors speculated that the Lactobacillus species could influence lipid metabolism through effects on bile acid metabolism and thus contributed to the risk for fatty liver [31]. An increased population of Parabacteroides, a major producer of succinate, was shown to be positively correlated with body weight in our study. To our knowledge, the link between abundance of these bacteria in response to diet change and liver status has not been reported so far. This genus could be interesting to be studied in HFDassociated metabolic disease such as NAFLD.

Finally, several limitations of our study should be mentioned. First, the generalizability of our findings may be limited owing to the low number of animals, the absence of food intake monitoring, and the lack of mechanistic approach. Also, it would be of great interest to test whether similar results would be obtained with different types of HFD.

In conclusion, we found that HFD leads to marked liver steatosis and that 1 week of $C D$ is sufficient to reverse steatosis. Furthermore, we observed that HFD changes microbiota and 1-week CD essentially reversed it. Together, these findings are consistent with the hypothesis that dietinduced changes to the gut microbiota may contribute to NAFLD. The question as to whether specific populations are responsible for weight gain and steatosis or are simply flourishing as a consequence of the diet being consumed (ie, are these populations a "cause" or an "effect") remains a key one. More broadly, our results emphasize that a more comprehensive understanding of diet-related diseases will benefit from elucidating links between nutrition, microbiome, and NAFLD. The use of germ-free mice and mice conventionalized with known microbial species should help to reveal the influence of specific populations on host health and in turn reveal therapeutic targets in the fight against obesity and associated liver diseases. 


\section{Acknowledgment}

This work was funded by the Förderung der wissenschaftlichen Forschung (FWF) (W 1226, DK Metabolic and Cardiovascular Disease) at the Medical University of Graz. We are grateful to the INRA MIGALE bioinformatics platform (http://migale.jouy.inra.fr/) for providing computational resources. We are also thankful to Ulrike Fackelmann from Medical University of Graz for technical assistance. The authors declare no conflict of interest. The funders had no role in the design of the study; in the collection, analyses, or interpretation of data; in the writing of the manuscript; or in the decision to publish the results. The author contributions are as follows: conceptualization, $\mathrm{KZ}$ and ZS; methodology, $\mathrm{KZ}$ and ZS; investigation, ZS and PMA; software, MM, KK, and MM; writing - original draft, ZS; writing - review and editing, ZS, PG, and KZ; funding acquisition, $\mathrm{KZ}$ and ZS; resources, PG and $\mathrm{KZ}$; supervision, $\mathrm{PG}$ and $\mathrm{KZ}$.

\section{R E F E R E N C E S}

[1] Brunt EM, Kleiner DE, Wilson LA, Belt P, Neuschwander-Tetri BA, Network NCR. Nonalcoholic fatty liver disease (NAFLD) activity score and the histopathologic diagnosis in NAFLD: distinct clinicopathologic meanings. Hepatology 2011;53: 810-20. https://doi.org/10.1002/hep.24127.

[2] Tilg H, Moschen AR. Evolution of inflammation in nonalcoholic fatty liver disease: the multiple parallel hits hypothesis. Hepatology 2010;52:1836-46. https://doi.org/10.1002/hep. 24001.

[3] Gaggini M, Morelli M, Buzzigoli E, DeFronzo RA, Bugianesi E, Gastaldelli A. Non-alcoholic fatty liver disease (NAFLD) and its connection with insulin resistance, dyslipidemia, atherosclerosis and coronary heart disease. Nutrients 2013;5: 1544-60. https://doi.org/10.3390/nu5051544.

[4] Safari Z, Gerard P. The links between the gut microbiome and non-alcoholic fatty liver disease (NAFLD). Cell Mol Life Sci 2019; 76:1541-58. https://doi.org/10.1007/s00018-019-03011-w.

[5] Drenick EJ, Fisler J, Johnson D. Hepatic steatosis after intestinal bypass-prevention and reversal by metronidazole, irrespective of protein-calorie malnutrition. Gastroenterology 1982;82:535-48. https://doi.org/10.1016/S0016-5085(82) 80403-4.

[6] Wigg AJ, Roberts-Thomson IC, Dymock RB, McCarthy PJ, Grose RH, Cummins AG. The role of small intestinal bacterial overgrowth, intestinal permeability, endotoxaemia, and tumour necrosis factor alpha in the pathogenesis of nonalcoholic steatohepatitis. Gut 2001;48:206-11.

[7] Henao-Mejia J, Elinav E, Jin C, Hao L, Mehal WZ, Strowig T, et al. Inflammasome-mediated dysbiosis regulates progression of NAFLD and obesity. Nature 2012;482:179-85. https:// doi.org/10.1038/nature10809.

[8] Schneider KM, Mohs A, Kilic K, Candels LS, Elfers C, Bennek E, et al. Intestinal microbiota protects against MCD dietinduced steatohepatitis. Int J Mol Sci 2019:20. https://doi.org/ 10.3390/ijms20020308.

[9] Le Roy T, Llopis M, Lepage P, Bruneau A, Rabot S, Bevilacqua $C$, et al. Intestinal microbiota determines development of non-alcoholic fatty liver disease in mice. Gut 2013;62:1787-94. https://doi.org/10.1136/gutjnl-2012-303816.

[10] Alkhouri N, Dixon LJ, Feldstein AE. Lipotoxicity in nonalcoholic fatty liver disease: not all lipids are created equal.
Expert Rev Gastroenterol Hepatol 2009;3:445-51. https://doi. org/10.1586/egh.09.32.

[11] Mells JE, Fu PP, Kumar P, Smith T, Karpen SJ, Anania FA. Saturated fat and cholesterol are critical to inducing murine metabolic syndrome with robust nonalcoholic steatohepatitis. J Nutr Biochem 2015;26:285-92. https://doi. org/10.1016/j.jnutbio.2014.11.002.

[12] Puri P, Baillie RA, Wiest MM, Mirshahi F, Choudhury J, Cheung $\mathrm{O}$, et al. A lipidomic analysis of nonalcoholic fatty liver disease. Hepatology 2007;46:1081-90. https://doi.org/10.1002/ hep.21763.

[13] Wiernsperger N. Hepatic function and the cardiometabolic syndrome. Diabetes Metab Syndr Obes 2013;6:379-88. https:// doi.org/10.2147/dmso.S51145.

[14] Lieber CS, Leo MA, Mak KM, Xu Y, Cao Q, Ren C, et al. Model of nonalcoholic steatohepatitis. Am J Clin Nutr 2004;79:502-9. https://doi.org/10.1093/ajcn/79.3.502.

[15] Eccleston HB, Andringa KK, Betancourt AM, King AL, Mantena SK, Swain TM, et al. Chronic exposure to a high-fat diet induces hepatic steatosis, impairs nitric oxide bioavailability, and modifies the mitochondrial proteome in mice. Antioxid Redox Signal 2011;15:447-59. https://doi.org/10. 1089/ars.2010.3395.

[16] O'Neill S, O’Driscoll L. Metabolic syndrome: a closer look at the growing epidemic and its associated pathologies. Obes Rev 2014;16:1-12. https://doi.org/10.1111/obr.12229.

[17] Després J-P, Lemieux I. Abdominal obesity and metabolic syndrome. Nature 2006;444:881. https://doi.org/10.1038/ nature05488.

[18] James AM, Collins Y, Logan A, Murphy MP. Mitochondrial oxidative stress and the metabolic syndrome. Trends Endocrinol Metab 2012;23:429-34. https://doi.org/10.1016/j. tem.2012.06.008.

[19] Than NN, Newsome PN. A concise review of non-alcoholic fatty liver disease. Atherosclerosis 2015;239:192-202. https:// doi.org/10.1016/j.atherosclerosis.2015.01.001.

[20] Daniel H, Gholami AM, Berry D, Desmarchelier C, Hahne H, Loh $G$, et al. High-fat diet alters gut microbiota physiology in mice. ISME J 2013;8:295. https://doi.org/10.1038/ismej.2013.155.

[21] Korem T, Zeevi D, Suez J, Weinberger A, Avnit-Sagi T, PompanLotan $\mathrm{M}$, et al. Growth dynamics of gut microbiota in health and disease inferred from single metagenomic samples. Science 2015;349:1101-6. https://doi.org/10.1126/science.aac4812.

[22] Turnbaugh PJ, Ridaura VK, Faith JJ, Rey FE, Knight R, Gordon JI. The effect of diet on the human gut microbiome: a metagenomic analysis in humanized gnotobiotic mice. Sci Transl Med 2009;1:6ra14. https://doi.org/10.1126/ scitranslmed.3000322.

[23] Ley RE, Turnbaugh PJ, Klein S, Gordon JI. Microbial ecology: human gut microbes associated with obesity. Nature 2006; 444:1022-3. https://doi.org/10.1038/4441022a.

[24] Turnbaugh PJ, Ley RE, Mahowald MA, Magrini V, Mardis ER, Gordon JI. An obesity-associated gut microbiome with increased capacity for energy harvest. Nature 2006;444: 1027-31. https://doi.org/10.1038/nature05414.

[25] Gart E, Souto Lima E, Schuren F, de Ruiter CGF, Attema J, Verschuren L, et al. Diet-independent correlations between bacteria and dysfunction of gut, adipose tissue, and liver: a comprehensive microbiota analysis in feces and mucosa of the ileum and colon in obese mice with NAFLD. Int J Mol Sci 2018;20. https://doi.org/10.3390/ijms20010001.

[26] Bojsen-Møller KN, Dirksen C, Jørgensen NB, Jacobsen SH, Serup AK, Albers PH, et al. Early enhancements of hepatic and later of peripheral insulin sensitivity combined with increased postprandial insulin secretion contribute to improved glycemic control after Roux-en-Y gastric bypass. Diabetes 2014;63:1725. https://doi.org/10.2337/db13-1307.

[27] Kowalski GM, Hamley S, Selathurai A, Kloehn J, De Souza DP, O'Callaghan S, et al. Reversing diet-induced metabolic 
dysregulation by diet switching leads to altered hepatic de novo lipogenesis and glycerolipid synthesis. Sci Rep 2016;6: 27541. https://doi.org/10.1038/srep27541.

[28] Sathananthan M, Shah M, Edens KL, Grothe KB, Piccinini F, Farrugia LP, et al. Six and 12 weeks of caloric restriction increases beta cell function and lowers fasting and postprandial glucose concentrations in people with type 2 diabetes. J Nutr 2015;145:2046-51. https://doi.org/10.3945/jn. 115.210617.

[29] Thaiss CA, Itav S, Rothschild D, Meijer MT, Levy M, Moresi C, et al. Persistent microbiome alterations modulate the rate of post-dieting weight regain. Nature 2016;540:544. https://doi. org/10.1038/nature20796.

[30] Spencer MD, Hamp TJ, Reid RW, Fischer LM, Zeisel SH, Fodor AA. Association between composition of the human gastrointestinal microbiome and development of fatty liver with choline deficiency. Gastroenterology 2011;140:976-86. https:// doi.org/10.1053/j.gastro.2010.11.049.

[31] Zeng H, Liu J, Jackson MI, Zhao FQ Yan L, Combs Jr GF. Fatty liver accompanies an increase in lactobacillus species in the hind gut of C57BL/6 mice fed a high-fat diet. J Nutr 2013;143: 627-31. https://doi.org/10.3945/jn.112.172460.

[32] Tiniakos DG, Vos MB, Brunt EM. Nonalcoholic fatty liver disease: pathology and pathogenesis. Annu Rev Pathol 2010;5:145-71. https://doi.org/10.1146/annurev-pathol-121808-102132.

[33] Folch J, Lees M, Sloane Stanley GH. A simple method for the isolation and purification of total lipides from animal tissues. J Biol Chem 1957;226:497-509.

[34] Sinha R, Abu-Ali G, Vogtmann E, Fodor AA, Ren B, Amir A, et al. Assessment of variation in microbial community amplicon sequencing by the microbiome quality control (MBQC) project consortium. Nat Biotechnol 2017;35:1077-86. https://doi.org/10.1038/nbt.3981.

[35] Schmieder R, Edwards R. Fast identification and removal of sequence contamination from genomic and metagenomic datasets. PLoS One 2011;6:e17288. https://doi.org/10.1371/ journal.pone.0017288.

[36] Bragg L, Stone G, Imelfort M, Hugenholtz P, Tyson GW. Fast, accurate error-correction of amplicon pyrosequences using Acacia. Nat Methods 2012;9:425-6. https://doi.org/10.1038/ nmeth.1990.

[37] Edgar RC. Search and clustering orders of magnitude faster than BLAST. Bioinformatics 2010;26:2460-1. https://doi.org/ 10.1093/bioinformatics/btq461.

[38] Caporaso JG, Kuczynski J, Stombaugh J, Bittinger K, Bushman FD, Costello EK, et al. QIIME allows analysis of highthroughput community sequencing data. Nat Methods 2010; 7:335-6. https://doi.org/10.1038/nmeth.f.303.

[39] Xu Z, Knight R. Dietary effects on human gut microbiome diversity. Br J Nutr 2015;113:S1-5. https://doi.org/10.1017/ S0007114514004127.

[40] Wu GD, Chen J, Hoffmann C, Bittinger K, Chen YY, Keilbaugh $\mathrm{SA}$, et al. Linking long-term dietary patterns with gut microbial enterotypes. Science 2011;334:105-8. https://doi. org/10.1126/science.1208344.

[41] Carmody RN, Gerber GK, Luevano Jr JM, Gatti DM, Somes L, Svenson KL, et al. Diet dominates host genotype in shaping the murine gut microbiota. Cell Host Microbe 2015;17:72-84. https://doi.org/10.1016/j.chom.2014.11.010.

[42] De Filippo C, Cavalieri D, Di Paola M, Ramazzotti M, Poullet JB, Massart S, et al. Impact of diet in shaping gut microbiota revealed by a comparative study in children from Europe and rural Africa. Proc Natl Acad Sci U S A 2010;107:14691-6. https://doi.org/10.1073/pnas.1005963107.

[43] Le Chatelier E, Nielsen T, Qin J, Prifti E, Hildebrand F, Falony $\mathrm{G}$, et al. Richness of human gut microbiome correlates with metabolic markers. Nature 2013;500:541. https://doi.org/10. 1038/nature12506.
[44] Riaz Rajoka MS, Shi J, Mehwish HM, Zhu J, Li Q, Shao D, et al. Interaction between diet composition and gut microbiota and its impact on gastrointestinal tract health. Food Sci Human Wellness 2017;6:121-30. https://doi.org/10.1016/j.fshw.2017.07.003.

[45] Bajaj JS, Ridlon JM, Hylemon PB, Thacker LR, Heuman DM, Smith $\mathrm{S}$, et al. Linkage of gut microbiome with cognition in hepatic encephalopathy. Am J Physiol Gastrointest Liver Physiol 2012; 302:G168-75. https://doi.org/10.1152/ajpgi.00190.2011.

[46] Wang B, Jiang X, Cao M, Ge J, Bao Q, Tang L, et al. Altered fecal microbiota correlates with liver biochemistry in nonobese patients with non-alcoholic fatty liver disease. Sci Rep 2016;6: 32002. https://doi.org/10.1038/srep32002.

[47] Cani PD, Amar J, Iglesias MA, Poggi M, Knauf C, Bastelica D, et al. Metabolic endotoxemia initiates obesity and insulin resistance. Diabetes 2007;56:1761-72. https://doi.org/10.2337/ db06-1491.

[48] Cani PD, Bibiloni R, Knauf C, Waget A, Neyrinck AM, Delzenne $\mathrm{NM}$, et al. Changes in gut microbiota control metabolic endotoxemia-induced inflammation in high-fat diet-induced obesity and diabetes in mice. Diabetes 2008;57:1470-81. https://doi.org/10.2337/db07-1403.

[49] Zhang C, Zhang M, Wang S, Han R, Cao Y, Hua W, et al. Interactions between gut microbiota, host genetics and diet relevant to development of metabolic syndromes in mice. ISME J 2010;4:232-41. https://doi.org/10.1038/ismej.2009.112.

[50] Heisel T, Montassier E, Johnson A, Al-Ghalith G, Lin Y-W, Wei $\mathrm{L}-\mathrm{N}$, et al. High-fat diet changes fungal microbiomes and interkingdom relationships in the murine gut. mSphere 2017; 2. https://doi.org/10.1128/mSphere.00351-17.

[51] Zhang C, Zhang M, Pang X, Zhao Y, Wang L, Zhao L. Structural resilience of the gut microbiota in adult mice under high-fat dietary perturbations. ISME J 2012;6:1848. https://doi.org/10. 1038/ismej.2012.27.

[52] Dethlefsen L, Relman DA. Incomplete recovery and individualized responses of the human distal gut microbiota to repeated antibiotic perturbation. Proc Natl Acad Sci U S A 2011;108(Suppl. 1):4554-61. https://doi.org/10.1073/pnas. 1000087107.

[53] Murphy EF, Cotter PD, Healy S, Marques TM, O’Sullivan O, Fouhy F, et al. Composition and energy harvesting capacity of the gut microbiota: relationship to diet, obesity and time in mouse models. Gut 2010;59:1635-42. https://doi.org/10.1136/ gut.2010.215665.

[54] Turnbaugh PJ, Backhed F, Fulton L, Gordon JI. Diet-induced obesity is linked to marked but reversible alterations in the mouse distal gut microbiome. Cell Host Microbe 2008;3: 213-23. https://doi.org/10.1016/j.chom.2008.02.015.

[55] Fleissner CK, Huebel N, Abd El-Bary MM, Loh G, Klaus S, Blaut $\mathrm{M}$. Absence of intestinal microbiota does not protect mice from diet-induced obesity. Br J Nutr 2010;104:919-29. https:// doi.org/10.1017/s0007114510001303.

[56] Zhang H, DiBaise JK, Zuccolo A, Kudrna D, Braidotti M, Yu Y, et al. Human gut microbiota in obesity and after gastric bypass. Proc Natl Acad Sci U S A 2009;106:2365-70. https://doi. org/10.1073/pnas.0812600106.

[57] DiBaise JK, Frank DN, Mathur R. Impact of the gut microbiota on the development of obesity: current concepts. Am J Gastroenterol Suppl 2012;1:22. https://doi.org/10.1038/ajgsup. 2012.5.

[58] Graham C, Mullen A, Whelan K. Obesity and the gastrointestinal microbiota: a review of associations and mechanisms. Nutr Rev 2015;73:376-85. https://doi.org/10.1093/ nutrit/nuv004.

[59] Elshaghabee FM, Bockelmann W, Meske D, de Vrese M, Walte HG, Schrezenmeir J, et al. Ethanol production by selected intestinal microorganisms and lactic acid bacteria growing under different nutritional conditions. Front Microbiol 2016;7: 47. https://doi.org/10.3389/fmicb.2016.00047. 
[60] Armougom F, Henry M, Vialettes B, Raccah D, Raoult D. Monitoring bacterial community of human gut microbiota reveals an increase in Lactobacillus in obese patients and methanogens in anorexic patients. PLoS One 2009;4:e7125. https://doi.org/10.1371/journal.pone.0007125.

[61] Kim KA, Gu W, Lee IA, Joh EH, Kim DH. High fat diet-induced gut microbiota exacerbates inflammation and obesity in mice via the TLR4 signaling pathway. PLoS One 2012;7:e47713. https://doi. org/10.1371/journal.pone.0047713.

[62] Moreira AP, Texeira TF, Ferreira AB, Peluzio Mdo C, Alfenas Rde C. Influence of a high-fat diet on gut microbiota, intestinal permeability and metabolic endotoxaemia. Br J Nutr 2012;108:801-9. https://doi.org/10.1017/ s0007114512001213.

[63] Cox LM, Yamanishi S, Sohn J, Alekseyenko AV, Leung JM, Cho I, et al. Altering the intestinal microbiota during a critical developmental window has lasting metabolic consequences. Cell 2014;158:705-21. https://doi.org/10.1016/j.cell.2014.05. 052.

[64] Kaakoush NO. Insights into the role of Erysipelotrichaceae in the human host. Front Cell Infect Microbiol 2015;5:84. https:// doi.org/10.3389/fcimb.2015.00084. 